\title{
UN MODELO DE EQUILIBRIO GENERAL DINÁMICO ESTOCÁSTICO ESTIMADO PARA LA ECONOMÍA COSTARRICENSE (2006-2014)
}

\section{A DYNAMIC STOCHASTIC GENERAL EQUILIBRIUM MODEL ESTIMATED FOR THE COSTA RICA ECONOMY (2006-2014)}

\section{Marco Vinicio Valerio Berrocal ${ }^{1}$}

\section{Resumen}

En este documento se presenta una especificación y estimación de un modelo dinámico estocástico de equilibrio general, métodos bayesianos que superan las críticas realizadas por Lucas (1976), Sims (1980) y Learmer (1991). Además, se realizan simulaciones de política monetaria.

Palabras Claves: Metas de inflación; Sector financiero; Estimación Bayesiana.

\begin{abstract}
This paper presents a specification and estimation of a dynamic stochastic general equilibrium model (DSGE), i.e. Bayesian methods that overcome the criticism by Lucas (1976), Sims (1980), and Learmer (1991). In addition, monetary policy simulations are conducted.
\end{abstract}

Keywords: Inflation Targeting; Finacial Sector; Bayesian Estimation.

\section{Introducción}

En este documento se presenta una especificación y estimación de un modelo dinámico estocástico de equilibrio general (DSGE), métodos bayesianos que superan las críticas realizadas por Lucas (1976), Sims (1980) y Learmer (1991). Además, se realizan simulaciones de política

doi: http://dx.doi.org/10.15359/eys.21-50.2

Fecha de recepción: 01-07-16. Fecha de reenvío: 10-10-16/23-10-16. Fecha de aceptación: 13-10-16. Fecha de publicación: 25-10-16.

${ }^{1}$ Licenciado en Economía, Universidad Nacional, Costa Rica. Funcionario, Instituto de Desarrollo Rural, Costa Rica. Correo electrónico: markxsw@gmail.com

Marco Vinicio Valerio Berrocal

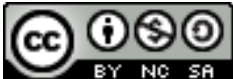

Revista Economía y Sociedad by Universidad Nacional is licensed under a CreativeCommons Reconocimiento-NoComercial- 
monetaria. Este modelo fue y puede ser utilizado para el análisis de la política monetaria ${ }^{2}$ en la economía costarricense.

La Metodología de Equilibrio General Dinámico en Costa Rica es poco utilizada en investigaciones macroeconómicas aplicadas a la de Costa Rica; pero, ha demostrado ser efectiva en el ámbito internacional Christiano, Trabant, \& Walentin, (2010) realizan una síntesis de los modelos DSGE y su aplicación en la política económica, Galí, Monacelli (2005) estudian la volatilidad del tipo de cambio en una economía pequeña y abierta, García y González (2007) estudian la efectividad de los mecanismos de transmisión de la política monetaria en países latinoamericanos, Liberati (2012) realiza un análisis del mecanismo de transmisión de la tasa de interés, Mohamed, Sales, (2015), Winkelried (2013) analiza el comportamiento del mercado de trabajo bajo imperfecciones en el mercado crediticio.

El presente documento consta de las siguientes secciones: en la segunda sección se explica la metodología de estimación, en la sección tercera se explican las ecuaciones del modelo, en la sección cuarta se muestran los resultados obtenidos del modelo y en la sección última se desarrollan conclusiones.

\section{Metodología de estimación}

El método para la estimación de los parámetros es el bayesiano. La visión bayesiana de la estimación econométrica incluye lo que los expertos llaman una visión a priori, es decir, su análisis se fundamenta en creencias previas del investigador ${ }^{3}$ que enriquecen el análisis de los datos. Es decir, suponga un modelo de la forma representada por la ecuación (1), donde se asume que $\theta$ es una magnitud no fija expuesta a un nivel de incertidumbre, y se espera con cierta probabilidad que el valor de $\theta$ sea cercano a una magnitud definida por la teoría económica.

$$
Y_{t+1}=F\left(Y_{t}, X_{t}, \theta, \varepsilon_{t}\right)
$$

De esta forma, mediante la combinación de información macroeconómica y conocimiento de la teoría económica, se pueden estimar los parámetros (distribución de probabilidades asociada a los parámetros), con la utilización del teorema de Bayes, de manera que:

\footnotetext{
${ }^{2}$ La política económica es la acción deliberada (de la autoridad o Gobierno) de modificar variables instrumento para alcanzar objetivos deseados (Tinbergen, 1956). Los objetivos puros de la política económica son: el crecimiento económico, el pleno empleo, la estabilidad de precios, el equilibrio de la balanza de pagos, y una distribución equitativa de la renta (Cuadrado, 2006). Adicionalmente, las políticas económicas pueden ser agrupadas según su taxonomía, refiriéndose a su capacidad de clasificarse en función del carácter de los instrumentos, su nivel de actuación y en su dimensión temporal; esto es: política fiscal o monetaria, microeconómica o macroeconómica, y de corto o largo plazo.

${ }^{3}$ Estas creencias se basan en la teoría economía nueva keynesiana, y los desarrollos resientes en modelación macroeconómica que en muchos casos dan valores esperados a nivel teórico de elasticidades.
}

2

Marco Vinicio Valerio Berrocal

(c) (i) 8 (O)

Revista Economía y Sociedad by Universidad Nacional is licensed under a CreativeCommons Reconocimiento-NoComercial- 


$$
\pi(\theta \mid x)=\frac{f(x \mid \theta) \pi(\theta)}{f(x)}
$$

Donde

$\pi(\theta \mid x)$; es la distribución de probabilidad a posteriori de los parámetros.

$\pi(\theta)$; es la Distribución a priori de los parámetros.

$f(x \mid \theta)$; densidad de muestreo.

$f(x)=\int f(x \mid \theta) \pi(\theta) d \theta$; es la función de verosimilitud marginal.

La distribución a posteriori es la distribución de probabilidad asociada a los valores de los parámetros estimados, la distribución a priori representan las creencias del investigador y de la teoría económica acerca del valor de los parámetros. La densidad de muestreo describe el condicional de los datos dados por parámetros. La función de verosimilitud marginal es una constante por lo que no agrega información adicional a la estimación, de manera que permite expresar la distribución posteriori en la forma de una proporción:

$$
\pi(\theta \mid x) \propto f(x \mid \theta) \pi(\theta)
$$

La función de verosimilitud es igual a la densidad de muestreo;

$$
\mathcal{L}(\theta ; x)=p(x \mid \theta)
$$

Debido a esto se considera que la densidad posterior es proporcional al producto de la función de verosimilitud y la densidad a priori;

$$
\pi(\theta \mid x) \propto \mathcal{L}(\theta ; x) \pi(\theta)
$$

Escude $(\underline{2010})$ considera este producto como la densidad a prior no normalizada que se denominará como $K(\theta \mid x)$. Al aplicar los logaritmos se obtiene la siguiente función:

$$
\operatorname{Máx}_{\theta} \ln K(\theta \mid x) \propto \ln \mathcal{L}(\theta ; x)+\ln \pi(\theta)
$$

Se puede inferir, por lo tanto, que el método bayesiano es una extensión del método de estimación de máxima verosimilitud ${ }^{4}$, y estos dos métodos pueden llegar a ser equivalentes si la distribución de probabilidad prior es una distribución uniforme ${ }^{5}$.

\footnotetext{
${ }^{4}$ Es importante mencionar que existen otros métodos de estimación, como el método generalizado de momentos (GMM), o el método generalizado de momentos simulados (Simulated GG). Se realiza la comparación máxima verosimilitud, ya que es un método ampliamente utilizado.

${ }^{5}$ Por lo que utilizar distribuciones uniformes equivale al caso de la estimación sin restricciones.
} 


\section{Medición de errores de especificación}

El estudio de los errores de especificación en modelos DSGE es un trabajo pionero de Del Negro et al. (2007). Este procedimiento establece una comparación de un modelo DSGE y un modelo VAR sin restricciones, en donde se analizan las diferencias entre funciones de impulso-respuesta de ambos modelos.

Se utiliza $\theta$ (prior del modelo DSGE) para obtener los priors de la varianza y parámetros un modelo VAR, la precisión de los priors del modelo VAR son multiplicados por un factor de escala llamado lamda $(\lambda)$. Este parámetro genera una familia de modelos que son llamados DSGE-VAR $\lambda)$; en el caso que $\lambda=0$ la estimación obtenida es equivalente a un modelo VAR estimado mediante el método de Mínimos Cuadrados Ordinarios; a medida que el valor de $\lambda$ tiende a infinito, se observa la densidad de probabilidad y si existe un aumento del logaritmo de verosimilitud con respecto al modelo DSGE-VAR $(\lambda=0)$, entonces los priors no ayudan a explicar el comportamiento de los datos.

Lo ideal, desde el punto de vista de una buena especificación del modelo, es que al aumentar el valor de $\lambda$, la densidad marginal no cambie sistemáticamente y que al final de la serie exista una mejora en la verosimilitud del modelo ${ }^{6}$.

\section{Tratamiento de datos}

La muestra de los datos de la investigación abarca el periodo de 2006-2014, que representa el periodo de transición ${ }^{7}$ de Metas de Inflación ${ }^{8}$. Los datos utilizados son de periodicidad trimestral. Las simulaciones y pronósticos se realizan en el periodo 2015-2017.

El software utilizado para la estimación del modelo es Matlab R2010a en conjunto con el módulo DYNARE (Dynamic Rational Expectations), diseñado para estimar y solucionar modelos dinámicos con perturbaciones aleatorias y expectativas racionales.

\footnotetext{
${ }^{6}$ Este criterio puede ser sensible a la elección de la muestra (Del Negro et al., 2007).

${ }^{7}$ En Costa Rica el régimen de política monetaria se encuentra en transición a un régimen de Metas de Inflación (El esquema no es oficial), bajo este esquema el control de la inflación se define como fin último; al obtener una inflación baja y estable la economía encuentra las condiciones necesarias para minimizar las pérdidas.

8 Para lograr el éxito de este esquema la inflación objetivo del Banco Central es comunicada formalmente a los agentes económicos, los cuales llevan periódicamente el pulso al cumplimiento del objetivo mediante una comunicación constante de la política monetaria. En síntesis, el régimen de política monetaria se basa en anclar las expectativas de inflación de los agentes a la meta propuesta por el Banco Central, para que los agentes anclen sus expectativas de inflación a la meta es necesario que el Banco Central forme credibilidad, para que esto suceda es imperativo que se cumplan algunas condiciones, tales como: la trasparencia, la rendición de cuentas, la publicación periódica de los planes y objetivos del Banco Central.
}

4

Marco Vinicio Valerio Berrocal

(c) (i) 80

Revista Economía y Sociedad by Universidad Nacional is licensed under a CreativeCommons Reconocimiento-NoComercial- 
Las series de tiempo no son tratadas con filtros de estacionalidad debido a que se puede eliminar información importante para la estimación. Esto concuerda con la posición de Hansen y Sargent (1993) que defiende el hecho de que los agentes toman decisiones sobre datos sin desestacionalizar, por esta razón remover la estacionalidad implica una pérdida de información importante que sesga las estimaciones de los parámetros.

Las variables observables utilizadas en la estimación del modelo, su fuente y su transformación, se detallan a continuación:

- Inflación $(\boldsymbol{\pi})$ : desviación absoluta con respecto a la tendencia Holdrick-Prescott de la Variación Trimestral Anualizada del Índice de Precios al Consumidor - Banco Central de Costa Rica/INEC (Lamda 2250)

- Brecha del Producto $(x)$ : ciclo Holdrick Prescott del PIB tendencia Ciclo - Banco Central de Costa Rica (Lamda 2250),

- Tasa de Interés Préstamos $\left(\boldsymbol{r}^{l}\right)$ : desviación absoluta con respecto a la tendencia Holdrick-Prescott de la Tasa de interés Activa - Banco Central de Costa Rica (Lamda 2250),

- Tasa de Interés Depósitos $\left(\boldsymbol{r}^{\boldsymbol{d}}\right)$ : desviación absoluta con respecto a la tendencia Holdrick-Prescott de la Tasa de Política Monetaria - Banco Central de Costa Rica (Lamda 2250),

- Variación Tipo de Cambio en Términos Americanos (e): desviación porcentual con respecto a la tendencia Holdrick-Prescott del promedio Compra - Venta del Tipo de cambio de Referencia - Banco Central de Costa Rica (Lamda 2250),

- Tasa de Interés Economía Externa $\left(\boldsymbol{r}^{\boldsymbol{e x}}\right)$ : desviación absoluta con respecto a la tendencia Holdrick-Prescott de la Tasa de interés efectiva a 6 meses de la Reserva Federal de los Estados Unidos - Reserva Federal de los Estados Unidos (Lamda 1600),

- Brecha del Crédito $(\boldsymbol{l})$ : ciclo Holdrick Prescott del Crédito del sistema financiero al sector privado por actividad económica - Banco Central de Costa Rica (Lamda 2250),

- Salarios $(\boldsymbol{w})$ : desviación porcentual con respecto a la tendencia Holdrick-Prescott del índice de Salarios Mínimos Nominales - Banco Central de Costa Rica (Lamda 2250),

\section{Modelo}

En la siguiente sección se describe el modelo Keynesiano ${ }^{10}$ que es estimado para la economía de Costa Rica, el cual contará con características propias de la economía nacional, estas

\footnotetext{
${ }^{9}$ Valor de Lamda del filtro definido en 2250 por Segura, C. \& Vásquez, J. (2011).

10 Es importante mencionar que el modelo se circunscribe en la síntesis nuevo keynesiana, que no debe ser confundida con los modelos Keynesianos "puros" y los modelos Neokeynesianos.
} 
características son: rigideces en los precios de los bienes (Calvo Pricing ${ }^{11}$ ), competencia monopolística, fricciones en el mercado financiero ("Search and Matching Frictions", Nash Bargain), un sistema financiero moderadamente concentrado, una economía pequeña y abierta al comercio, un régimen monetario enfocado en la estabilidad macroeconómica (del cual el principal y único instrumento es la Tasa de interés de Política Monetaria), traspaso imperfecto del tipo de cambio y de la Tasa de Interés de Política Monetaria (TPM), rigideces en la fijación de salarios y hábitos en el consumo.

\section{Hogares}

El hogar representativo en la economía tiene como objetivo maximizar su utilidad, la maximización de la utilidad determina la cantidad de consumo y trabajo que este efectúa. La función de utilidad del hogar representativo puede tener una especificación que no es aditivamente separable en términos intertemporales.

La especificación intertemporal hace referencia a la formación de hábitos de consumo. Los hábitos de consumo consisten en el hecho de que los agentes se acostumbran a un nivel relativo de utilidad que depende del consumo realizado en el periodo anterior, por lo que resiste (o tarda) en modificar su canasta de consumo. Esto se debe a una característica interna del ser humano, que le supone un costo hacer frente a los cambios en su entorno. La función de utilidad con hábitos de consumo se modela de la siguiente forma:

$$
\text { Máx } E_{0} \sum_{t=0}^{\infty} \beta^{t}\left(\frac{1}{1-\sigma}\left(\frac{C_{t}}{C_{t-1}} C_{t-1}^{(1-\chi)}\right)^{1-\sigma}-\frac{N_{t}^{1+\phi}}{1+\phi}\right)
$$

Donde el $\mathrm{C}_{\mathrm{t}}$ es el consumo y $\mathrm{N}_{\mathrm{t}}$ representa las horas trabajadas, y los parámetros $\sigma$ y $\phi$ son interpretados como la elasticidad intertemporal del consumo y la desutilidad del trabajo respectivamente, además el parámetro $\beta$ es un factor de descuento intertemporal o de impaciencia. La función de utilidad tiene la propiedad de ser isoelástica, este tipo de función es llamada "función de utilidad con aversión relativa al riesgo constante". El parámetro $\chi$ es el grado de persistencia intertemporal d la variable consumo.

Siguiendo a Galí (2005) en una pequeña economía abierta, el término de consumo es una composición entre $C_{h, t}$ y $C_{e, t}$ que representan el consumo de bienes locales y bienes extranjeros, la composición es realizada por un agregador CES (Elasticidad Sustitución Constante), de manera el que consumo agregado es una función de definida como:

11 "Calvo Pricing" o fijación de precios alá calco hace referencia a un esquema de fijación de precios propuesto por Guillermo Calvo (1983). En este esquema las empresas cambian una fracción de los precios con cierta probabilidad durante un periodo determinado, generalmente de un año.

6

Marco Vinicio Valerio Berrocal

(c) (P) 8 (2)

Revista Economía y Sociedad by Universidad Nacional is licensed under a CreativeCommons Reconocimiento-NoComercial- 


$$
C_{t}=\left[(1-\alpha)^{\frac{1}{\eta}} C_{h, t}^{\frac{\eta-1}{\eta}}+(\alpha)^{\frac{1}{\eta}} C_{e, t}^{\frac{\eta-1}{\eta}}\right]^{\frac{\eta}{\eta-1}}
$$

De manera que $\eta$ representa la elasticidad sustitución entre bienes locales y extranjeros.

Para cada tipo de bien, local como extranjero, existe "i" bienes en un intervalo continuo de $[0,1]$, de forma que las cantidades de bienes consumidos de cada tipo son terminados por la siguiente función CES:

$$
C_{h, t}=\left(\int_{0}^{1} C_{h, t}(i)^{\frac{\varepsilon-1}{\varepsilon}} d i\right)^{\frac{\varepsilon}{\varepsilon-1}} ; \quad C_{e, t}=\left(\int_{0}^{1} C_{e, t}(i)^{\frac{\varepsilon-1}{\varepsilon}} d i\right)^{\frac{\varepsilon}{\varepsilon-1}}
$$

Donde $\varepsilon$ representa la elasticidad sustitución entre cada bien i contenido en $[0,1]$.

La maximización de la función esta entonces sujeta a la siguiente restricción presupuestaria:

$$
\int_{0}^{1}\left[P(i)_{h, t} C(i)_{h, t}+P(i)_{e, t} C(i)_{e, t}\right] d i+E_{t}\left(Q_{t+1} D_{t+1}\right) \leq D_{t}+W_{t} N_{t}+T_{t}
$$

Donde $P(i)_{h, t}$ y $P(i)_{e, t}$ denotan los precios de los i bienes domésticos y extranjeros respectivamente. La asignación óptima del gasto de consumo produce las siguientes funciones de demanda:

$$
C(i)_{h, t}=\left(\frac{P(i)_{h, t}}{P_{h, t}}\right)^{-\varepsilon} C_{h, t} ; \quad C(i)_{e, t}=\left(\frac{P(i)_{e, t}}{P_{h, t}}\right)^{-\varepsilon} C_{e, t}
$$

Para todo " $i$ " que pertenece a el intervalo [0,1] $P_{h, t}=\left(\int_{0}^{1} P_{h, t}(i)^{\varepsilon-1} d i\right)^{\frac{1}{\varepsilon-1}}$ y $P_{e, t}=$ $\left(\int_{0}^{1} P_{e, t}(i)^{\varepsilon-1} d i\right)^{\frac{1}{\varepsilon-1}}$ son los precios agregados de cada tipo de bien, por lo que el gasto óptimo de consumo entre cada tipo de bien viene dado por las funciones;

$$
C_{h, t}=(1-\alpha)\left(\frac{P_{h, t}}{P_{t}}\right)^{-\eta} C_{t} ; \quad C_{e, t}=\alpha\left(\frac{P_{h, t}}{P_{t}}\right)^{-\eta} C_{t}
$$

Donde $\mathrm{P}_{\mathrm{t}}=\left[(1-\alpha) \mathrm{P}_{\mathrm{h}, \mathrm{t}}{ }^{\mathrm{n}-1}+\alpha \mathrm{P}_{\mathrm{e}, \mathrm{t}^{n-1}}\right]^{\frac{1}{n-1}}$ es el IPC, por lo que el parámetro $\alpha$ es interpretado como el grado de apertura de la economía, o bien como la ponderación de relativa de los bienes externos dentro del IPC.

Tomando en cuenta las condiciones óptimas expuestas anteriormente, se puede expresar la restricción presupuestaria de la forma:

$$
\mathrm{P}_{\mathrm{t}} \mathrm{C}_{\mathrm{t}}+\mathrm{E}_{\mathrm{t}}\left(\mathrm{Q}_{\mathrm{t}+1} \mathrm{D}_{\mathrm{t}+1}\right) \leq \mathrm{D}_{\mathrm{t}}+\mathrm{W}_{\mathrm{t}} \mathrm{N}_{\mathrm{t}}+\mathrm{T}_{\mathrm{t}}+\Pi_{t}^{B}+\Pi_{t}^{F}
$$

Marco Vinicio Valerio Berrocal

\section{(c) (i) (2)}

Revista Economía y Sociedad by Universidad Nacional is licensed under a CreativeCommons Reconocimiento-NoComercial- 
Que muestra las cantidades de consumo y los precios de forma agregada.

Donde $Q_{t+1}$ es un factor de descuento estocástico que mantiene una relación directa con la tasa de interés ${ }^{12}$ de la economía. La variable $\mathrm{T}_{\mathrm{t}}$ representa una transferencia gubernamental de suma fija, la variable $\Pi_{t}^{B}$ representa el beneficio obtenido por los bancos, la variable $\Pi_{t}^{F}$ representa el beneficio obtenido por las empresas, por último $\mathrm{W}_{\mathrm{t}}$ son los salarios percibidos por los hogares como resultado de su trabajo en las empresas.

La maximización de la ecuación 7 esta entonces sujeta a la siguiente restricción presupuestaria representada por la ecuación 13 de manera que las condiciones de primer orden son:

$$
\begin{gathered}
\beta R^{d}{ }_{t} E_{t}\left\{\left(\frac{\left(\frac{C_{t}}{C_{t-1}^{X}}\right)}{\left(\frac{C_{t+1}}{C_{t}^{x}}\right)}\right)^{\sigma} \frac{P_{t+1}}{P_{t}}\right\}=1 \\
\left(\frac{C_{t}}{C_{t-1}^{X}}\right)^{\sigma} N_{t}^{\phi}=\frac{W_{t}}{P_{t}}
\end{gathered}
$$

Las ecuaciones solución del Lagrangiano son nombradas en la literatura como la ecuación de Euler y la oferta de trabajo, respectivamente. La ecuación de Euler caracteriza la dinámica del consumo dentro del modelo esta es, por definición, una condición intertemporal que implica que los agentes económicos pueden suavizar la trayectoria del consumo a lo largo del tiempo, esto al modificar las cantidades de consumo futuro y consumo presente.

\section{Empresas}

En esta economía existen dos tipos de empresas, las empresas productoras de bienes intermedios y las empresas de bienes finales.

Las empresas productoras de bienes intermedios se encuentran en un mercado competitivo, tanto de factores como de bienes. Cada una de las empresas representativas de la economía produce con una función de producción Cobb Douglas con rendimientos decrecientes a escala de forma: $Y_{t}=e^{a_{t}} N_{t}{ }^{1-\alpha^{\prime}}$; donde $Y_{t}$ representa la cantidad producida del bien intermedio, $N_{t}$ representa la cantidad de horas trabajadas en la producción del bien intermedio y $\mathrm{a}_{\mathrm{t}}$ es un shock tecnológico que sigue un proceso autorregresivo de orden 1, como muestra la ecuación (16).

$$
a_{t}=\rho a_{t-1}+u_{t} ; \quad u_{t} \sim N\left(0, \sigma_{u}^{2}\right)
$$

\footnotetext{
12 Siguiendo a Ljungqvist y Sargent (2000) $Q_{t}=R_{t}^{d-1} ; R_{t}{ }^{d}=\left(1+r_{t}\right)$, donde $r_{t}$ es la tasa de interés de la economía. La variable $Q_{t}$ también es conocido como "Debreu Price" en un esquema "Sequential trading" de Arrow. 8 
Las empresas de bienes finales toman el bien intermedio producido por las empresas de bienes intermedios $\mathrm{y}$ venden bienes diferenciados a los hogares en un mercado de competencia monopolística. Se supone, además, que las variaciones de los precios del bien final ocurren con una probabilidad $1-\omega$, de acuerdo con Calvo (1983).

Las empresas de bienes finales fijarán sus nuevos precios $\mathrm{P}_{\mathrm{t}}{ }_{\mathrm{t}}$ con el fin de maximizar su utilidad esperada, con la restricción que les impone la demanda del bien final, de manera que:

$$
\begin{gathered}
\operatorname{máx}_{\bar{P}_{\mathrm{H}, \mathrm{t}}} \sum_{\mathrm{k}=0}^{\infty}(\omega \beta)^{\mathrm{k}} \mathrm{E}_{\mathrm{t}}\left\{\frac{\lambda_{\mathrm{t}+\mathrm{k}}}{\lambda_{\mathrm{t}}}\left[\frac{\mathrm{P}_{\mathrm{H}, \mathrm{t}}^{*}}{\mathrm{P}_{\mathrm{H}, \mathrm{t}+\mathrm{k}}}-m c_{\mathrm{t}+1}\right] Y(\mathrm{i})_{\mathrm{h}, \mathrm{t}+\mathrm{k}}\right\} \\
\text { st. } \mathrm{Y}(\mathrm{i})_{\mathrm{h}, \mathrm{t}}=\left(\frac{\mathrm{P}(\mathrm{i})_{\mathrm{h}, \mathrm{t}}}{\mathrm{P}_{\mathrm{h}, \mathrm{t}}}\right)^{-\varepsilon} \mathrm{Y}_{\mathrm{h}, \mathrm{t}}
\end{gathered}
$$

La solución de este problema de maximización brinda la ecuación de determinación de los precios:

$$
\frac{P(i)_{h, t}}{P_{h, t}}=\frac{\varepsilon}{\varepsilon-1} \frac{E_{t} \sum_{t=0}^{\infty}(\omega \beta)^{k} m c_{t+k}\left(\frac{P_{h, t+1}}{P_{h, t}}\right)^{\varepsilon} C_{t+k}^{1-\sigma}}{E_{t} \sum_{t=0}^{\infty}(\omega \beta)^{k}\left(\frac{P_{h, t+1}}{P_{h, t}}\right)^{\varepsilon} C_{t+k}^{1-\sigma}}
$$

Esta ecuación es loglinearizada para obtener la Curva de Phillips Neo keynesiana:

$$
\begin{gathered}
\pi_{\mathrm{h}, \mathrm{t}}=\omega\left(\mathrm{mc}_{\mathrm{t}}\right)+\beta E\left(\pi_{\mathrm{h}, \mathrm{t}+1}\right)+\varepsilon_{\mathrm{t}} \\
\theta=\frac{(1-\omega)(1-\omega \beta)}{\omega} \\
\varepsilon_{\mathrm{t}}=\frac{\mathrm{u}_{\mathrm{t}}}{\omega}
\end{gathered}
$$

Las rigideces salariales permiten diferenciar dos medidas de producción, el nivel de producción de la economía y el nivel de producción en ausencia de rigideces de precios. Este último es equivalente al producto potencial, lo que permite modelar la curva IS utilizando la brecha del producto:

$$
\widehat{y_{t}}=y_{t}-y_{t}^{q f}
$$

\section{Autoridad monetaria}

La política de la autoridad monetaria sigue una regla tipo Taylor (1993), la cual consiste en la fijación de la tasa de interés con el objetivo de minimizar la diferencia de la inflación con 
respecto a la meta de inflación, esto permite modelar el comportamiento de una Autoridad Monetaria que tiene como principal objetivo lograr una inflación baja y estable, lo que corresponde a un esquema de Metas de Inflación. La regla de Taylor además incluye la brecha del producto, este componente modela el interés de manejar una política contra cíclica, es decir un objetivo subsidiario de estabilizar los ciclos económicos en pos de un crecimiento económico estable.

En la línea de investigación de Muñoz y Tenorio (2008) se adopta una Regla de Taylor Suavizada (Ecuación 24), esta especificación de la Regla de Taylor introduce un valor rezagado en la TPM que indica la existencia de cierto nivel de inercia ${ }^{13}$ en las modificaciones de la tasa interés de política monetaria, que es interpretado como activismo en la ejecución de política.

$$
r_{t}^{\text {pol }}=\Omega r_{t-1}^{\text {pol }}+(1-\Omega)\left(-\log (\beta)+E\left(\pi_{t+1}\right)+\phi\left(E\left(\widehat{\pi}_{t+1}\right)-\pi_{t}^{M}\right)\right)
$$

\section{Sistema bancario}

En un modelo con sistema financiero explícito existen dos tasas de interés: una para depósitos definida por la política de la Autoridad Monetaria ${ }^{14}$, y otra para los préstamos definida por el sector financiero. El diferencial de ambas tasas de interés es el margen de intermediación financiera. El sector financiero maximizará sus beneficios incrementando el margen de intermediación hasta donde la demanda por préstamos se lo permita. El modelo propuesto por Liberati (2012) supone la existencia de rigideces en el mercado financiero como restricciones de búsqueda y pareo (Search and Matching), esquemas de negociación de precios Nash y un grado de concentración Bancaria. El saldo de líneas de créditos $L_{t}^{B}$ sigue una ley de movimiento definida por:

$$
L_{t}^{B}=\left(1-\rho^{B}\right) L_{t-1}^{B}+q_{t}^{B} V_{t}^{B}
$$

Donde $\rho^{\mathrm{B}}$ es la probabilidad de que ocurra una separación entre uno de estos pareos, y $\mathrm{q}_{\mathrm{t}}^{\mathrm{B}}$ es la probabilidad de llenar una línea de un crédito, y $V_{t}^{B}$ representa las líneas de crédito disponibles. También se define a $s_{t}^{B}$ como la proporción del número de empresas que buscan un crédito, de

\footnotetext{
13 También se interpreta $\Omega$ como el activismo de la autoridad monetaria para realizar política. Es decir, cuando $\Omega$ tiende a 1 , el Banco Central no realiza política, y en caso contrario si $\Omega$ tiende 0 , esto refleja un completo activismo. ${ }^{14}$ La tasa de interés promedio (ponderado) pagada por los depósitos en el sistema financiero es la Tasa de Interés Básica Pasiva (TBP), en el modelo se intenta modelar el efecto de la Política Monetaria sobre la economía ante esta situación se elige la TPM como tasa de interés de depósitos y no la TBP. Motivos adicionales motivaron esta decisión son: a) el traspaso y correlación TPM- TBP es alto, lo que hace trivial calcular el traspaso TPM-TBP-Tasa Activa (TA). b) La TPM es mejor indicador de la política monetaria. c) La TA se relaciona de manera muy estrecha con la inversión y los ciclos económicos.
}

10

Marco Vinicio Valerio Berrocal

(c) (i) 80

Revista Economía y Sociedad by Universidad Nacional is licensed under a CreativeCommons Reconocimiento-NoComercial- 
manera que sigue una ley de movimiento determinada por las empresas que separaron de una línea de crédito.

$$
\mathrm{s}_{\mathrm{t}}^{\mathrm{B}}=1-\left(1-\rho^{\mathrm{B}}\right) \mathrm{L}_{\mathrm{t}-1}^{\mathrm{B}}
$$

Además $\mathrm{p}_{\mathrm{t}}^{\mathrm{B}}$ se define como la probabilidad de que una empresa consiga una línea de crédito ofertada por un banco, por último, se define la estrechez del mercado financiero como $\theta_{t}^{c}=$ $\frac{s_{t}^{F}}{V_{t}^{F}}$.

El banco, al inicio de cada periodo, recibe depósitos y una capitalización $\mathrm{X}_{\mathrm{t}}=M_{t}-M_{t-1}$, de los hogares y el Banco Central, respectivamente. Al mismo tiempo utiliza este dinero para financiar préstamos y pagar los costos de generar nuevas líneas de crédito; por lo que para todo periodo se cumple la identidad:

$$
\frac{D_{t}}{P_{t}}+\frac{X_{t}}{P_{t}}=\frac{L_{t}}{P_{t}}+k^{B} V_{t}^{B}
$$

Donde, $\frac{L_{t}}{P_{t}}$ el monto a financiar es equivalente al necesitado por las empresas para ejecutar sus operaciones:

$$
\frac{L_{t}}{P_{t}}=\mathrm{w}_{\mathrm{t}} \mathrm{N}_{\mathrm{t}} \mathrm{L}_{\mathrm{t}}^{\mathrm{B}}
$$

Al final del periodo los bancos pagan a sus depositantes la cantidad:

$$
R_{t}^{d} \frac{D_{t}}{P_{t}}=R_{t}^{d}\left(w_{t} N_{t} L_{t}^{B}+k^{B} V_{t}^{B}-\frac{X_{t}}{P_{t}}\right)
$$

Utilizando la identidad del Margen de intermediación financiero, la ecuación de beneficios del Banco;

$$
\mathrm{R}_{\mathrm{t}}^{\mathrm{L}} \frac{L_{t}}{P_{t}}-\mathrm{R}_{\mathrm{t}}^{\mathrm{d}} \frac{D_{t}}{P_{t}}
$$

El banco representativo va maximizar el valor descontado de sus ganancias, de manera que:

$$
\begin{gathered}
\mathrm{J}_{\mathrm{t}}^{\mathrm{B}}=\max \left[\left(\mathrm{R}_{\mathrm{t}}^{\mathrm{L}}-\mathrm{R}_{\mathrm{t}}^{\mathrm{d}}\right) \mathrm{w}_{\mathrm{t}} \mathrm{N}_{\mathrm{t}} \mathrm{L}_{\mathrm{t}}^{\mathrm{B}}-\mathrm{R}_{\mathrm{t}}^{\mathrm{d}} \mathrm{k}^{\mathrm{B}} \mathrm{V}_{\mathrm{t}}^{\mathrm{B}}+\mathrm{R}_{\mathrm{t}}^{\mathrm{d}} \frac{\mathrm{X}_{\mathrm{t}}}{\mathrm{P}_{\mathrm{t}}}+\beta \mathrm{E}_{\mathrm{t}}\left(\frac{\lambda_{\mathrm{t}+1}}{\lambda_{\mathrm{t}}}\right) \mathrm{J}_{\mathrm{t}+1}^{\mathrm{B}}\right] \\
\text { st. } \quad L_{t}^{B}=\left(1-\rho^{B}\right) L_{t-1}^{B}+q_{t}^{B} V_{t}^{B}
\end{gathered}
$$

Donde $\mathrm{k}^{\mathrm{B}}$ refleja el costo real de generar una nueva línea de crédito y $\frac{\mathrm{X}_{t}}{\mathrm{P}_{\mathrm{t}}}$ se define como $\frac{\mathrm{M}_{\mathrm{t}}-\mathrm{M}_{\mathrm{t}-1}}{\mathrm{P}_{t}}$ que son las inyecciones monetarias que realice el Banco Central que están en función de su posición en el mercado de liquidez, así como del costo del dinero definido por la TPM. 
La condición de primer orden que soluciona el problema al que se enfrenta los bancos es interpretada como "la condición de creación de créditos" corresponde a la ecuación ${ }^{15}$ :

$$
\frac{R_{t}^{d} k^{B}}{\mathrm{q}_{t}^{B}}=\left(R_{t}^{L}-R_{t}^{d}\right) w_{t} N_{t} L_{t}^{B}+\left(1-\rho^{B}\right) \beta \mathrm{E}_{\mathrm{t}}\left(\frac{\lambda_{\mathrm{t}+1}}{\lambda_{\mathrm{t}}}\right) \frac{R_{t+1}^{d} k^{B}}{\mathrm{q}_{t+1}^{B}}
$$

La condición para ofrecer una nueva línea de crédito depende del flujo descontado de los ingresos del banco y del ahorro sobre la probabilidad que una empresa llene una línea de crédito $\mathrm{q}_{t}^{B}$. En particular, el costo esperado de financiar una línea de crédito, $\frac{R_{t}^{d} k^{B}}{\mathrm{q}_{t}^{B}}$, es igual al ingreso marginal que el banco obtiene de un préstamo realizado a una empresa. Adicionalmente, nótese que en el caso de que el costo real de generar una línea de crédito sea igual a cero $R_{t}^{L}=R_{t}^{d}$.

La tasa de interés de los préstamos $R_{t}^{L}$ es determinada mediante la maximización de un producto de Nash entre los excedentes de los bancos y las empresas:

$$
\max \left(S_{t}^{F}\right)^{z}\left(S_{t}^{B}\right)^{1-z}
$$

Donde el parámetro $z$ representa el poder de negociación de cada uno de los bancos, y $S_{t}^{F}$ junto con $S_{t}^{B}$ representan los excedentes de las empresas y los bancos respectivamente. Se tiene que el excedente de las empresas es igual a:

$$
S_{t}^{F}=\frac{\mathrm{Y}_{\mathrm{t}}}{\mu_{\mathrm{t}}}-\mathrm{w}_{\mathrm{t}} \mathrm{R}_{\mathrm{t}}^{\mathrm{L}} \mathrm{N}_{\mathrm{t}}+\left(1-\rho^{B}\right) \beta E_{t}\left(\frac{\lambda_{t+1}}{\lambda_{t}}\right)\left(1-p_{t+1}^{B}\right) S_{t+1}^{F}
$$

y el excedente de los bancos es igual a:

$$
S_{t}^{B}=\frac{R_{t}^{d} k^{B}}{\mathrm{q}_{t}^{B}}
$$

La solución de la maximización del producto de Nash da como resultado la ecuación que determina la tasa de interés de los préstamos, esta ecuación viene dada por:

$$
R_{t}^{L}=\frac{1-\mathrm{z}}{\mathrm{w}_{\mathrm{t}} \mathrm{N}_{\mathrm{t}}} \frac{\mathrm{Y}_{\mathrm{t}}}{\mu_{\mathrm{t}}}+\frac{\mathrm{z}}{\mathrm{w}_{\mathrm{t}} \mathrm{N}_{\mathrm{t}}}\left[\mathrm{w}_{\mathrm{t}} \mathrm{R}_{\mathrm{t}}^{\mathrm{d}} \mathrm{N}_{\mathrm{t}}-\left(1-\rho^{B}\right) \beta E_{t}\left(\frac{\lambda_{t+1}}{\lambda_{t}}\right) \frac{R_{t+1}^{d} k^{B}}{\theta_{t+1}^{c}}\right]
$$

Lo que indica que la tasa de interés de los préstamos es un promedio ponderado entre las ganancias de las empresas, de la diferencia entre de los ahorros presentes en los bancos y tasa

\footnotetext{
${ }^{15}$ Se utiliza el teorema de la envolvente, siguiendo el desarrollo de Liberati (2012). 12 
de interés sobre los depósitos netos esperados, resultado de mantener una línea de crédito con una empresa. Los ponderadores son determinados por el poder de negociación de los agentes.

\section{Sector externo}

Este apartado muestra las relaciones de la economía con el resto del mundo, este segmento se basa principalmente en los trabajos de Galí y Monacellí (2002) y Galí (2008).

\section{Identidades}

Parafraseando a Galí y Monacelli (2002) se definirán diversas identidades, las cuales serán utilizadas al determinar el equilibrio de la economía con el resto del mundo.

En primer lugar, se define al IPC como un promedio ponderado de los precios domésticos y los precios extranjeros, de manera que:

$$
\mathrm{p}_{\mathrm{t}}=(1-\alpha) \mathrm{p}_{\mathrm{H}, \mathrm{t}}+\alpha \mathrm{p}_{\mathrm{F}, \mathrm{t}}
$$

Donde al loglinealizar alrededor del estado estacionario con $\mathrm{p}_{\mathrm{H}, \mathrm{t}}=\mathrm{p}_{\mathrm{F}, \mathrm{t}} \mathrm{y}$ definiendo los términos de intercambio como $s_{t}=p_{H, t}-p_{F, t}$ se puede escribir la ecuación (38) como:

$$
\mathrm{p}_{\mathrm{t}}=\mathrm{p}_{\mathrm{H}, \mathrm{t}}+\alpha \mathrm{s}_{\mathrm{t}}
$$

Aplicando primeras diferencias a los componentes de la ecuación, se obtiene que las variaciones de la inflación son resultado de las variaciones de los precios domésticos y de los términos de intercambio.

$$
\pi_{\mathrm{t}}=\pi_{\mathrm{H}, \mathrm{t}}+\alpha \Delta \mathrm{s}_{\mathrm{t}}
$$

La variación de la inflación es proporcional a las variaciones de los precios de los bienes producidos domésticamente, y son un porcentaje $\alpha$ de las variaciones de los términos de intercambio; el parámetro $\alpha$ es interpretado como el índice de apertura de la economía. Bajo el supuesto de una pequeña economía abierta, el índice de precios externos es igual al nivel de precios del mundo por lo que tiene la identidad $\pi_{\mathrm{F}, \mathrm{t}}=\pi_{\mathrm{t}}{ }^{*}$.

La identidad correspondiente al cumplimiento de la Ley de un solo precio, la paridad del poder de compra, permite escribir los términos de intercambio de la siguiente forma:

$$
\mathrm{s}_{\mathrm{t}}=\Delta \mathrm{e}_{\mathrm{t}}-\mathrm{p}_{\mathrm{H}, \mathrm{t}}+\mathrm{p}_{\mathrm{t}}^{*}
$$

La ecuación loglinearizada del tipo de cambio real viene dada por $\mathrm{q}_{t}=\mathrm{s}_{\mathrm{t}}-\mathrm{p}_{\mathrm{H}, \mathrm{t}}-\mathrm{p}_{\mathrm{t}}$, por lo que combinando las ecuaciones (41) y la identidad del tipo de cambio real, se obtiene: 


$$
\mathrm{q}_{\mathrm{t}}=(1-\alpha) \mathrm{s}_{\mathrm{t}}
$$

Ahora bien, se asume traspaso incompleto del tipo de cambio, por lo que se incorpora el término $\psi_{\mathrm{t}}$ a la ecuación (42) y finalmente se obtiene la ecuación incorporada al modelo:

$$
\mathrm{q}_{\mathrm{t}}=\psi_{\mathrm{t}}+(1-\alpha) \mathrm{s}_{\mathrm{t}}
$$

Donde $\psi_{\mathrm{t}}$ refleja los desvíos de la ley de un solo precio, esta brecha es definida por $\psi_{\mathrm{t}}=$ $\Delta \mathrm{e}_{\mathrm{t}}+\mathrm{p} *_{\mathrm{t}}-\mathrm{p}_{\mathrm{t}}$.

\section{Intercambio Internacional de Riesgos}

En la línea de investigación de Galí (2002), se asumen mercados internacionales de valores completos (Risk Pooling) (Imen Ben Mohamed, Marine Sales, 2015). La condición de primer orden del problema del consumidor de la economía externa viene dada por:

$$
\beta \mathrm{R}^{*}{ }_{\mathrm{t}} \mathrm{E}_{\mathrm{t}}\left\{\left(\frac{\left(\frac{\mathrm{C}_{\mathrm{t}}{ }^{*}}{\mathrm{C}_{\mathrm{t}-1}^{\chi}{ }^{*}}\right)}{\left(\frac{\mathrm{C}_{\mathrm{t}+1}{ }^{*}}{\mathrm{C}_{\mathrm{t}}^{\chi^{*}}}\right)}\right)^{\sigma} \frac{\mathrm{P}_{\mathrm{t}+1}{ }^{*}}{\mathrm{P}_{\mathrm{t}}{ }^{*}}\right\}=1
$$

Combinando la condición de primer orden de la economía doméstica y la economía extrajera, se obtiene la relación entre consumo de ambas economías para todos los periodos, $\mathrm{C}_{\mathrm{t}}=\vartheta \mathrm{C}_{\mathrm{t}}{ }^{*} \mathcal{Q}_{\mathrm{t}}^{\frac{1}{\sigma}}$, adicionalmente, se aplican logaritmos a la relación y se aplica el supuesto de traspaso imperfecto del tipo de cambio, para obtener la ecuación:

$$
c_{t}-\chi c_{t-1}=c_{t}^{*}-\chi c_{t-1}^{*}+\left(\frac{1-\alpha}{\sigma}\right)\left(s_{t}+\psi_{t}\right)
$$

Esta ecuación refleja que los niveles de consumo de ambas economías son determinados por los términos de intercambio en la magnitud $\left(\frac{1-\alpha}{\sigma}\right)$; es decir, si la variable $s_{t}$ incrementa la economía nacional, podrá incrementar su consumo.

\section{Paridad del interés y términos de intercambio}

La relación de paridad de interés descubierta se escribe como la relación existente entre las variaciones del tipo de cambio y el diferencial de tasa de interés, sin contemplar ninguna prima de riesgo a priori. Así que esta relación es representada por la siguiente ecuación. 


$$
\mathrm{E}_{\mathrm{t}}\left\{\mathrm{Q}_{\mathrm{t}, \mathrm{t}+1}\left[\mathrm{R}_{\mathrm{t}}^{\mathrm{d}}-\mathrm{R}_{\mathrm{t}}^{*}\left(\frac{\mathcal{E}_{\mathrm{t}+1}}{\mathcal{E}_{\mathrm{t}}}\right)\right]\right\}=0
$$

Loglinearizando se obtiene:

$$
r_{t}{ }^{d}-r_{t}^{*}=E_{t}\left(\Delta e_{t+1}\right)
$$

Combinado la condición anterior con los términos de intercambio:

$$
s_{t}=\left(r_{t}^{*}-E_{t}\left\{\pi_{t+1}^{*}\right\}\right)-\left(r_{t}^{d}-E_{t}\left\{\pi_{H, t+1}\right\}\right)+E_{t}\left\{s_{t+1}\right\}
$$

Este arreglo permite representar las variaciones de los términos de intercambio como divergencias entre la condición de paridad en términos reales y, como ya se mencionó, estas variaciones tendrán un impacto en el nivel de consumo de la economía nacional.

\section{Generación de expectativas}

Las expectativas son determinantes del comportamiento económico, con información perfecta es de esperarse que las expectativas sean certeras y se materialicen en las variables observables; es decir que $X_{t}^{e}=E\left(X_{t+1}\right)$. Winkelried (2013) propone la existencia de rigideces en la información que los agentes disponen (y pueden procesar) en cada momento, esta condición implica la posibilidad que en el corto plazo $X_{t}^{e} \neq E\left(X_{t+1}\right)$, en este escenario es posible que la expectativa converja al caso de información completa a medida que los agentes aprendan de sus errores.

La ecuación de generación de expectativas es:

$$
\pi_{t}^{e}=\rho^{e} \pi_{t-1}^{e}+\left(1-\rho^{e}\right) E\left(\pi_{t+1}\right)+\varepsilon^{\pi}{ }_{t}
$$

Donde el parámetro $\rho^{e}$ depende de la velocidad con la que los agentes aprenden de sus errores; en el caso que $\rho^{e}=0$, las expectativas son racionales con un ajuste $\sin$ fricciones. El tiempo en periodos que los agentes tardan en ajustar sus expectativas es $\rho^{e} /\left(1-\rho^{e}\right)$.

\section{Negociaciones salariales}

La lógica de ajuste de los salarios sigue el esquema de Calvo (1983), en este caso el problema de los hogares es el de maximizar el valor intertemporal descontado de los salarios, esto sujeto a la demanda efectiva por trabajo a la que se enfrentan. De manera que el problema de optimización de los hogares se representa con las siguientes ecuaciones: 


$$
\begin{gathered}
\operatorname{máx}_{w} \sum_{\mathrm{k}=0}^{\infty}\left(\omega_{W} \beta\right)^{\mathrm{k}} \mathrm{E}_{\mathrm{t}}\left\{\frac{1}{1-\sigma}\left(\frac{\mathrm{C}_{\mathrm{t}}}{\mathrm{C}_{\mathrm{t}-1+\mathrm{k}}} \mathrm{C}_{\mathrm{t}-1+\mathrm{k}}^{(1-\mathrm{x})}\right)^{1-\sigma}-\frac{\mathrm{N}_{\mathrm{t}+\mathrm{k}}^{1+\phi}}{1+\phi}\right\} \\
\text { st. } \mathrm{N}(\mathrm{i})_{\mathrm{t}}=\left(\frac{\mathrm{W}(\mathrm{i})_{\mathrm{t}}}{\mathrm{W}_{\mathrm{t}}}\right)^{-\varepsilon_{w}} \mathrm{~N}_{\mathrm{h}, \mathrm{t}}
\end{gathered}
$$

\section{Resultados}

En este apartado se detallan los resultados y elementos considerados para ejecutar la estimación de los parámetros. La elección de priors se realiza considerando las investigaciones de Liberati (2012), García y González (2007), y las características de cada parámetro en cuestión.

Para estimar la persistencia de los choques y la varianza, se eligen distribuciones prior distribuciones uniformes y gamma inversa respectivamente, la elección de distribuciones uniformes es a razón de no existir información para determinar los priors de la persistencia de los choques. Se hace una excepción con el parámetro de la persistencia del choque de política monetaria p2; para este parámetro se elige una distribución beta con promedio 0,1 para evitar reacciones contrarias en la TPM. Woodford (2003) y, Clarida, Galí \& Gertler (1999) determinan que una perturbación de política monetaria muy persistente genera una respuesta inversa en el ajuste de tasa de interés, que es contraria la diversa evidencia empírica obtenida por medio de modelos VAR.

Los parámetros del bloque de la economía internacional son calibrados utilizando los valores estimados por Christiano et al. (2010), el cual estima los parámetros de un modelo DSGE para la economía de Estados Unidos. La magnitud de estos parámetros de presenta en el Tabla 1.

Tabla 1.

Parámetros calibrados del bloque externo

\begin{tabular}{cc}
\hline Parámetro & Magnitud \\
\hline$\beta^{*}$ & 0,99 \\
$\sigma^{*}$ & 1 \\
$\mathrm{~K}$ & 0,21 \\
$\chi^{*}$ & 0,76 \\
$\Omega^{*}$ & 0,87 \\
$\phi_{\pi}$ & 1,43 \\
$\phi_{\mathrm{y}}$ & 0,04 \\
\hline Fuente: Elaboración propia con base en Christiano et al. (2010)
\end{tabular}

16 
Economía y Sociedad, Vol. 21, № 50 julio-diciembre 2016, pp. 1-33

Tabla 2

Resumen de Interpretación de parámetros

\begin{tabular}{|c|c|c|c|}
\hline Parámetro & Interpretación & Parámetro & Interpretación \\
\hline$\beta$ & Factor de Impaciencia & $z$ & Poder de Negociación de los Bancos \\
\hline$\sigma$ & Elasticidad Utilidad del Consumo & $\rho$ & Activismo Politica Monetaria \\
\hline$\varphi$ & Des-utilidad del Trabajo & $\delta_{g}$ & Peso del Objetivo Inflación \\
\hline$x$ & Magnitud de Hábitos del Consumo & $\delta_{y}$ & Peso del Objetivo Producto \\
\hline$\alpha^{\prime}$ & Parámetro Tecnológico del Capital & $\delta_{e}$ & Peso del Objetivo Estabilidad Cambiaria \\
\hline$\alpha$ & Transables en el IPC & $\beta^{*}$ & Factor de Impaciencia Economia Externa ( $\left.E_{c} E_{x}\right)$ \\
\hline$\omega$ & $\begin{array}{c}\text { Probabilidad de Cambios en los } \\
\text { Precios }\end{array}$ & $\sigma^{*}$ & Elasticidad Utilidad del Consumo Ec.Ex \\
\hline$\varepsilon$ & Mark-Up & $\phi_{\pi}$ & Peso del Objetivo Inflación Ec.Ex \\
\hline$k^{B}$ & $\begin{array}{c}\text { Costo de Generar una Vacante } \\
\text { Crediticia }\end{array}$ & K & Parámetro curva de Phillips Ec.Ex \\
\hline$p^{6}$ & $\begin{array}{c}\text { Probabilidad Exógena de Cesar un } \\
\text { Crédito }\end{array}$ & $\Omega^{*}$ & Probabilidad de Calvo Ec.Ex \\
\hline$\mu$ & $\begin{array}{c}\text { Extemalidad Positiva "Liquid Market } \\
\text { Effect" }\end{array}$ & $\phi_{y}$ & Peso del Objetivo Producto Ec.Ex \\
\hline$\varepsilon_{w}$ & $\begin{array}{c}\text { Elasticidad Sustitución del Mercado } \\
\text { de Trabajo }\end{array}$ & $\chi^{*}$ & Magnitud de Hábitos del Consumo \\
\hline$p 1$ & Persistencia del choque Tecnológico & $p 5$ & Persistencia del choque del Canal Expectativas \\
\hline$p 2$ & $\begin{array}{c}\text { Persistencia del choque del Canal } \\
\text { TPM }\end{array}$ & $p 6$ & Persistencia del choque del Canal Crédito Amplio \\
\hline$p 3$ & $\begin{array}{c}\text { Persistencia del choque Canal Tipo } \\
\text { de Cambio }\end{array}$ & $p 7$ & $\begin{array}{c}\text { Persistencia del choque de la Economía } \\
\text { Internacional }\end{array}$ \\
\hline$p 4$ & $\begin{array}{l}\text { Persistencia del choque del Canal } \\
\text { Crédito Estrecho }\end{array}$ & $p 8$ & $\begin{array}{c}\text { Persigtencia del choque de Oferta Agregada - } \\
\text { Salariog }\end{array}$ \\
\hline
\end{tabular}

Fuente: Elaboración propia 
Tabla 3.

\section{Resultados de estimación Bayesiana}

\begin{tabular}{|c|c|c|c|c|c|c|}
\hline Parámetros & $\begin{array}{l}\text { Media } \\
\text { Prior }\end{array}$ & $\begin{array}{c}\text { Media } \\
\text { Posterior }\end{array}$ & $\begin{array}{c}\text { Limite Inferior } \\
90 \%\end{array}$ & $\begin{array}{c}\text { Limite Superior } \\
90 \%\end{array}$ & $\begin{array}{c}\text { Distribución } \\
\text { Prior }\end{array}$ & $\begin{array}{l}\text { Est. Desv. } \\
\text { Posterior }\end{array}$ \\
\hline$\sigma$ & 1,0000 & 2,6691 & 1,8132 & 3,7057 & Norm & 1,0000 \\
\hline$\varphi$ & 1,0000 & 0,9266 & 0,7911 & 1,0959 & Norm & 0,1000 \\
\hline$\alpha^{\prime}$ & 0,4400 & 0,4506 & 0,4416 & 0,4590 & Beta & 0,0100 \\
\hline$\beta$ & 0,9700 & 0,9837 & 0,9722 & 0,9981 & Beta & 0,0200 \\
\hline$\omega$ & 0,7500 & 0,6203 & 0,5576 & 0,6736 & Beta & 0,0500 \\
\hline$\rho$ & 0,8000 & 0,6864 & 0,5973 & 0,7690 & Beta & 0,1000 \\
\hline$\delta_{\pi}$ & 1,5000 & 1,4357 & 1,1984 & 1,6312 & Gamma & 0,2000 \\
\hline$\delta_{y}$ & 0,1250 & 0,1669 & 0,1331 & 0,2032 & Gamma & 0,0250 \\
\hline$\alpha$ & 0,4000 & 0,0639 & 0,0272 & 0,1081 & Beta & 0,1000 \\
\hline$p^{B}$ & 0,8000 & 0,9535 & 0,8640 & 1,0000 & Beta & 0,2500 \\
\hline z & 0,5000 & 0,4738 & 0,4510 & 0,4958 & Beta & 0,2500 \\
\hline$p 1$ & 0,5000 & 0,6917 & 0,1604 & 0,9996 & Uniforme & 0,2887 \\
\hline$p 2$ & 0,1000 & 0,1004 & 0,0892 & 0,1127 & Beta & 0,0100 \\
\hline$p 3$ & 0,5000 & 0,2656 & 0,0000 & 0,5666 & Uniforme & 0,2887 \\
\hline$p 4$ & 0,5000 & 0,9730 & 0,9437 & 0,9999 & Uniforme & 0,2887 \\
\hline$p 5$ & 0,5000 & 0,0574 & 0,0001 & 0,1238 & Uniforme & 0,2887 \\
\hline$p 6$ & 0,5000 & 0,5979 & 0,4318 & 0,7759 & Uniforme & 0,2887 \\
\hline$p 7$ & 0,1200 & 0,1233 & 0,1123 & 0,1320 & Beta & 0,0100 \\
\hline$p 8$ & 0,5000 & 0,9303 & 0,8783 & 0,9952 & Uniforme & 0,2887 \\
\hline$\mu^{\prime}$ & 0,5000 & 0,4983 & 0,4735 & 0,5219 & Beta & 0,0250 \\
\hline$\rho^{b}$ & 0,0750 & 0,0560 & 0,0383 & 0,0763 & Beta & 0,0250 \\
\hline$k^{B}$ & 0,0500 & 0,0267 & 0,0000 & 0,0868 & Gamma & 0,0500 \\
\hline$\rho_{\text {aprend }}$ & 0,8000 & 0,8823 & 0,8243 & 0,9326 & Beta & 0,0500 \\
\hline$x$ & 0,5000 & 0,0402 & 0,0000 & 0,0928 & Uniforme & 0,2887 \\
\hline Perturbaciones & $\begin{array}{c}\text { Media } \\
\text { Prior } \\
\end{array}$ & $\begin{array}{c}\text { Media } \\
\text { Posterior }\end{array}$ & $\begin{array}{c}\text { Limite Inferior } \\
90 \% \\
\end{array}$ & $\begin{array}{c}\text { Limite Superior } \\
90 \% \\
\end{array}$ & Prior & $\begin{array}{l}\text { Est. Desv. } \\
\text { Posterior }\end{array}$ \\
\hline Tecnológico & 1,0000 & 0,14400 & 0,11850 & 0,16790 & Inv. Gamma & Inf \\
\hline Canal TPM & 1,0000 & 0,13750 & 0,11860 & 0,15730 & Inv. Gamma & Inf \\
\hline $\begin{array}{c}\text { Canal Tipo de } \\
\text { Cambio }\end{array}$ & 1,0000 & 0,20420 & 0,16050 & 0,24330 & Inv. Gamma & Inf \\
\hline $\begin{array}{c}\text { Canal Crédito } \\
\text { Estrecho }\end{array}$ & 1,0000 & 0,14570 & 0,11870 & 0,16630 & Inv. Gamma & Inf \\
\hline $\begin{array}{c}\text { Canal } \\
\text { Expectativas }\end{array}$ & 1,0000 & 0,13750 & 0,11770 & 0,15570 & Inv. Gamma & Inf \\
\hline $\begin{array}{c}\text { Canal Crédito } \\
\text { Amplio }\end{array}$ & 1,0000 & 0,58700 & 0,23570 & 0,95430 & Inv. Gamma & Inf \\
\hline $\begin{array}{c}\text { Economia } \\
\text { Internacional }\end{array}$ & 1,0000 & 0,13640 & 0,11760 & 0,15610 & Inv. Gamma & Inf \\
\hline $\begin{array}{c}\text { Oferta Agregada } \\
\text {-Salarios }\end{array}$ & 1,0000 & 0,42550 & 0,21350 & 0,66150 & Inv. Gamma & Inf \\
\hline
\end{tabular}

Fuente: Elaboración propia

El parámetro de descuento o impaciencia estimado $\beta$ es de 0,9837 el cual es congruente con una tasa de interés anualizada del 6,79\%.

18

Marco Vinicio Valerio Berrocal

(cc) (i) 8 (9)

Revista Economía y Sociedad by Universidad Nacional is licensed under a CreativeCommons Reconocimiento-NoComercial-

Compartirlgual 4.0 Internacional License.

Creado a partir de la obra en http://www.revistas.una.ac.cr/index.php/economia 
El parámetro de activismo de política monetaria $(\rho)$ revela un menor activismo que el prior; por otra parte, el parámetro relacionado con el objetivo de producción $\left(\delta_{y}\right)$ es mayor al prior y el parámetro de objetivo de inflación $\left(\delta_{\pi}\right)$ es mayor al priors seleccionados, es importante resaltar que el relativo de los parámetros de política monetaria $\left(\delta_{\pi} / \delta_{y}\right)$ en el prior es de 11.53 y de la estimación posterior resulta un mayor peso al objetivo de producto ${ }^{16}$ con un relativo del 8.57 .

La estimación del parámetro de Calvo $(\omega)$, que indica rigidez en los precios, se estima en 0.6203 ; lo que implica que una empresa cambia sus precios cada 7 meses y 13 días, en promedio. Este valor es mayor a la evidencia encontrada en León y Valerio (2014), donde se estima, ponderando las duraciones promedio de los índices de precios de artículos regulados y no regulados, que el parámetro puede ser 0,11 aproximadamente, es diferencia se aduce a que en León y Valerio (2014) se calcula la duración promedio de cambio de índices de precios; los índices son una construcción lineal de los precios por lo que su variación es más probable, dando como resultado la estimación frecuencias de variación de precios menores ${ }^{17}$.

Las estimaciones muestran que el parámetro $\mathrm{k}^{\mathrm{B}}$ (el costo administrativo de crear una línea de crédito) es 0.0267; lo cual es un nivel bajo comparado con el nivel prior tomado de Liberati (2012). Además, el poder de negociación de los bancos se estima en 0.4738; lo que indica que el poder de negociación de los bancos es mayor al poder de negociación de los agentes; esto incide sobre la dinámica de ajuste de la tasa de interés activa, la hace más persistente ante variaciones de la TPM e incrementa el traspaso directo de la tasa de interés, sobre el indirecto.

\section{Diagnóstico mediante el Método Montecarlo con Cadenas de Markov}

El algoritmo de Hastings Metrópolis utiliza el Método Montecarlo y Cadenas de Markov para calcular las distribuciones de los parámetros, la validez de las estimaciones de los parámetros depende de la estabilidad del cadena de Markov, es decir, esta debe ser una cadena asintóticamente estacionaria y los resultados deben ser repetibles; las diferentes simulaciones de las cadenas no deben ser muy distintas entre ellas.

Con lo que respecta a la convergencia de cada cadena de Markov (Figura 1), se evidencia que no existen saltos significativos entre estados; hacia el final de la cadena, a partir de la iteración

\footnotetext{
16 La importancia relativa mostrada por el Banco Central por el objetivo de producción tiene como resultado: 1Una lenta convergencia al objetivo de inflación. 2- Crecimiento en el corto plazo sin un impacto estructural sobre el producto potencial. El Banco Central debe tomar en cuenta el sacrificio (Trade off) que existe entre el crecimiento económico y la inflación en el corto plazo, para maximizar el bienestar social.

17 La frecuencia en la se modifican los precios representada por el parámetro $\omega$, incide directamente sobre la efectividad de la política monetaria. La magnitud estimada de 0.6203 indica que las rigideces de precios existentes en la economía de Costa Rica son tales que permiten la existencia de la no neutralidad monetaria en el corto plazo, por lo tanto, la política monetaria es efectiva.
}

Marco Vinicio Valerio Berrocal

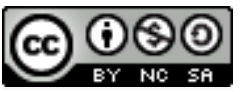

Revista Economía y Sociedad by Universidad Nacional is licensed under a CreativeCommons Reconocimiento-NoComercial- 
número 25.000, esto se hace más evidente debido a que se aprecia que esta adquiere un valor estable $^{18}$, es decir la cadena tiene un promedio (Interval) y varianza finitos $(\mathrm{M} 2)^{19}$.
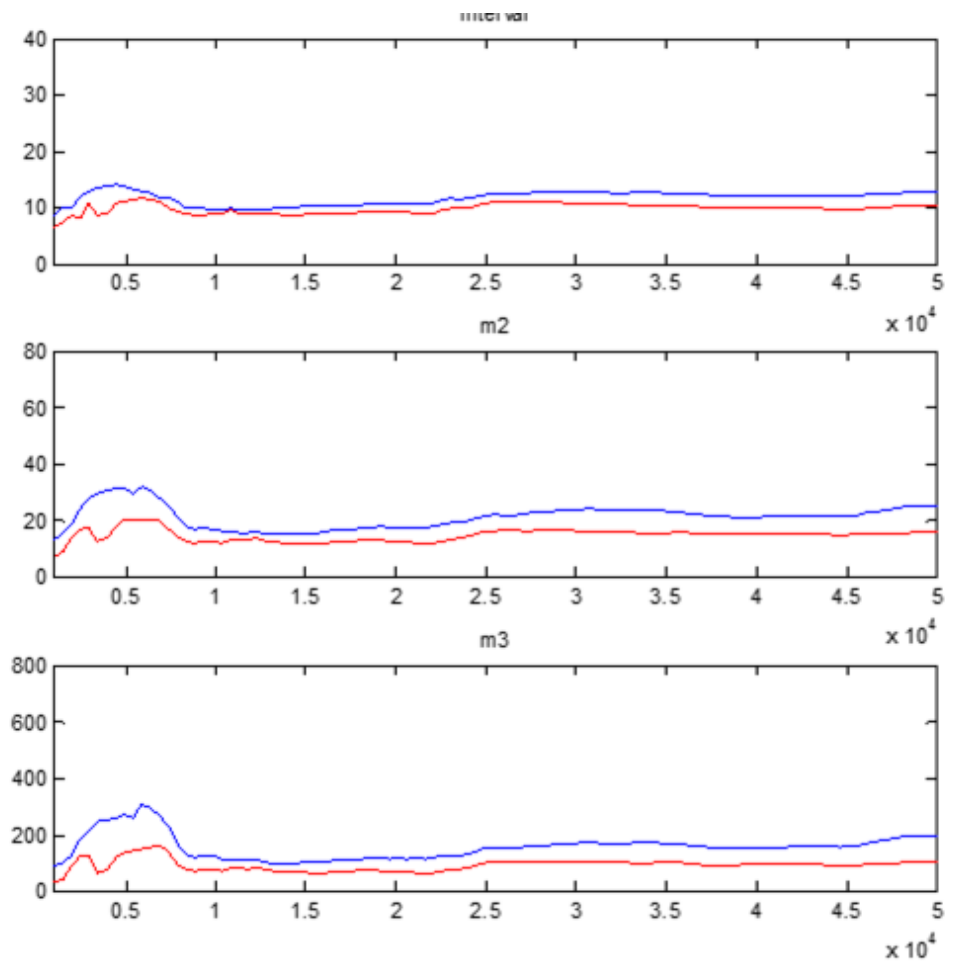

Nota: Iteraciones en el eje de las abscisas, valor de la cadena de Markov eje de las ordenadas

Figura 1. Diagnóstico Multivariado de la Cadena de Markov. Fuente: elaboración propia.

Al realizar la estimación Bayesiana de los parámetros se ejecuta el algoritmo de HastingMetropolis, utilizando 4 cadenas de Markov de 50.000 iteraciones (esto implica 200.000 iteraciones en la estimación de distribuciones posterior). El promedio entre las 4 cadenas es $29.72 \%$ con una desviación estándar de 5.83 p.p. entre cada cadena, lo que evidencia estabilidad entre los resultados de simulación de la cadena de Markov, validando la convergencia de las cadenas.

\section{Diagnóstico de la especificación del modelo}

La diferencia entre los logaritmos de verosimilitudes de los casos extremos de $\lambda=0$ y $\lambda \rightarrow \infty$ es positiva de magnitud 10.39; esto implica que al aumentar el peso de las restricciones impuestas

18 Estadísticamente se hace alusión en este caso al concepto de convergencia en el sentido de Cèsaro, veáse Dhrymes $(\underline{1970})$ p.389.

19 "Interval" es un estadístico construido alrededor de las medias de los parámetros. "M2" es un estadístico que representa la varianza. "M3" es un estadístico basado en terceros momentos. Todos deben ser convergentes.

20

Marco Vinicio Valerio Berrocal

(c) (i) 80

Revista Economía y Sociedad by Universidad Nacional is licensed under a CreativeCommons Reconocimiento-NoComercial- 
en el modelo DSGE (EI caso $\lambda \rightarrow \infty$ ), estas ayudan a explicar el comportamiento de la economía. Esto es evidencia a favor de una buena especificación en el modelo DSGE.

Tabla 4.

\section{Diagnóstico de Especificación Modelo DSGE}

\begin{tabular}{ll}
\hline Modelo & Verosimilitud \\
\hline Modelo DSGE-VAR $(\hat{\lambda}=9,5102)$ & $-365,2069$ \\
Modelo DSGE-VAR $(\lambda=0)$ & $\mathbf{- 3 7 5 , 6 0 5 5}$ \\
Modelo DSGE-VAR $(\lambda=1)$ & -332.0373 \\
Modelo DSGE-VAR $(\lambda=2)$ & $-347,9527$ \\
Modelo DSGE-VAR $(\lambda=5)$ & $-350,5982$ \\
Modelo DSGE-VAR $(\lambda=9)$ & $-368,6702$ \\
Modelo DSGE-VAR $(\lambda \rightarrow \infty)$ & $-\mathbf{3 6 5 , 5 4 5 1}$ \\
Modelo DSGE & $-395,8049$ \\
\hline
\end{tabular}

Fuente: elaboración propia.

\section{Simulaciones regímenes de política monetaria}

Se aproxima la función de pérdida del Banco Central por medio de la función de utilidad de los hogares, esta aproximación permite evaluar el efecto de diferentes políticas, o regímenes de política, sobre el bienestar ${ }^{20}$ de la sociedad.

Woodford (2001) deriva la función de pérdida social a partir de una función de utilidad de la forma:

$$
W_{t}=E_{t}\left\{\sum_{t=0}^{\infty} \beta^{t} U_{t}\right\}
$$

Donde $U_{t}$ es una función de utilidad instantánea que depende del consumo y del trabajo de los hogares, de manera que $U_{t}\left(C_{t}, N_{t}\right)$. La aproximación de Taylor de la función de utilidad alrededor del estado estacionario para el caso de la función de utilidad separable, es igual a:

$$
U_{t}-U \simeq U_{C} C\left(y_{t}+\frac{1-\sigma}{2} y_{t}^{2}\right)+U_{N} N\left(n_{t}+\frac{1+\varphi}{2} n_{t}^{2}\right)
$$

${ }^{20}$ Se define bienestar como la suma de utilidades de los individuos que integran la sociedad, siguiendo a John Hicks (1974). 
Desarrollando estos términos, se obtiene la aproximación de polinomios de Taylor de la función de utilidad, representada por la ecuación (7), la cual es a la vez la función de pérdida que el Banco Central desea minimizar ${ }^{21}$.

$$
\mathbb{W} \equiv-\frac{(1-\alpha)}{2} \sum_{t=0}^{\infty} \beta^{t}\left(\frac{\varepsilon}{\theta} \pi_{H, t}^{2}+\left(\sigma+\frac{\alpha^{\prime}+\varphi}{1-\alpha^{\prime}}\right) \hat{y}_{t}^{2}\right)+t . i . p+o\left(\|a\|^{3}\right)
$$

A partir de este desarrollo de la anterior ecuación es posible caracterizar la función de pérdida en función de varianzas, expresando la pérdida de una fracción del consumo de estado estacionario; lo que facilita la interpretación de las medidas de bienestar. Para el caso de un modelo con rigideces salariales y hábitos en el consumo:

$$
\mathbb{V} \equiv-\frac{(1-\alpha)}{2}\left(\frac{\varepsilon}{\theta} \operatorname{Var}\left(\pi_{t}^{p}\right)+\frac{\varepsilon_{w}\left(1-\alpha^{\prime}\right)}{\lambda_{w}} \operatorname{Var}\left(\pi_{t}^{w}\right)+\left(\sigma+\frac{\alpha^{\prime}+\varphi}{1-\alpha^{\prime}}\right)(1-\chi) \operatorname{Var}\left(\hat{y}_{t}\right)\right)
$$

Los parámetros son calibrados utilizando los resultados de la estimación Bayesiana, lo que da como resultado $\lambda_{w}=0,574 ; \theta=0,2365 ; \varphi=0,9266 ; \alpha=0,0636 ; \alpha^{\prime}=0,4506$ y suponiendo un "Mark-Up" de los precios, y salarios, sobre los costos marginales de las empresas del $11 \%$ (lo que implica que $\varepsilon=10, \varepsilon_{w}=10$ ). Se calculan los momentos teóricos de las variables utilizando una muestra de 2.000 periodos.

La línea base de la cual se comparan las simulaciones es el arreglo de metas de inflación al IPC resultante de la estimación Bayesiana en tal como lo muestra la ecuación (66).

$$
r_{t}^{d}=\rho r_{t-1}^{d}+(1-\rho)\left(\delta_{\pi} E\left(\pi_{t+1}\right)+\delta_{y} \hat{y}_{t}\right)
$$

Los escenarios alternativos simulados y sus respectivas reglas de política son los siguientes:

- Tipo de cambio fijo (o de variación constante)

$$
\Delta \mathrm{e}_{\mathrm{t}}=0
$$

Metas de inflación con administración de la volatilidad cambiaria

$$
r_{t}^{d}=\rho r_{t-1}^{d}+(1-\rho)\left(\delta_{\pi} E\left(\pi_{t+1}\right)+\delta_{y} \hat{y}_{t}+\delta_{e} \Delta e_{t}\right)
$$

\footnotetext{
${ }^{21}$ La demostración completa de este desarrollo matemático se encuentra en Woodford (2003) Cap. 6 "Inflation stabilization and Welfare". El caso de una pequeña economía abierta es abordado por Galí (2005) Cap. 7 “Monetary Policy and the Open Economy").
}

22

Marco Vinicio Valerio Berrocal

(c) (i) (2)

Revista Economía y Sociedad by Universidad Nacional is licensed under a CreativeCommons Reconocimiento-NoComercial- 
- Metas de inflación al componente doméstico de la inflación

$$
r_{t}^{d}=\rho r_{t-1}^{d}+(1-\rho)\left(\delta_{\pi} E\left(\pi_{H, t+1}\right)+\delta_{y} \hat{y}_{t}\right)
$$

- Metas de inflación puras

$$
r_{t}^{d}=\rho r_{t-1}^{d}+(1-\rho) \delta_{\pi} E\left(\pi_{t+1}\right)
$$

- Metas de inflación puras sin suavizar

$$
r_{t}^{d}=\delta_{\pi} E\left(\pi_{t+1}\right)
$$

Tabla 5.

\begin{tabular}{|c|c|c|c|c|c|c|c|}
\hline \multirow{2}{*}{ Escenario } & \multicolumn{3}{|c|}{ Varianzas (simuladas) } & \multicolumn{3}{|c|}{$\begin{array}{l}\text { Contribución a la pérdida de } \\
\text { bienestar }\end{array}$} & \multirow{2}{*}{ Total } \\
\hline & $\begin{array}{l}\text { Brecha del } \\
\text { producto }\end{array}$ & Inflación & Salarios & $\begin{array}{c}\text { Brecha del } \\
\text { producto }\end{array}$ & Inflación & Salarios & \\
\hline $\begin{array}{c}\text { Escenario base } \\
\text { (transición a metas de } \\
\text { inflación; estimación) }\end{array}$ & 0,9194 & 13,78 & 11,21 & -0.02 & -2.73 & -0.57 & $-3.32 \%$ \\
\hline Tipo de cambio fijo & 29,87 & 57,03 & 35,32 & -0.72 & -11.29 & -1.79 & $-13.80 \%$ \\
\hline $\begin{array}{c}\text { Flotación administrada } \\
\left(\delta_{e}=0,54\right)\end{array}$ & 1,20 & 13,95 & 11,55 & -0.03 & -2.76 & -0.59 & $-3.38 \%$ \\
\hline $\begin{array}{c}\text { Flotación administrada } \\
\left(\delta_{\theta}=1\right)\end{array}$ & 88,16 & 15,08 & 11,44 & -2.14 & -2.98 & -0.58 & $-5.70 \%$ \\
\hline $\begin{array}{c}\text { Metas inflación } \\
\text { (componente doméstico) }\end{array}$ & 0,90 & 13,69 & 11,04 & -0.02 & -2.71 & -0.56 & $-3.29 \%$ \\
\hline $\begin{array}{l}\text { Metas de inflación } \\
\text { "puras" (sin objetivo de } \\
\text { producto) }\end{array}$ & 0,96 & 13,49 & 10,77 & -0.02 & -2.67 & -0.55 & $-3.24 \%$ \\
\hline $\begin{array}{l}\text { Metas de inflación } \\
\text { "puras" sin suavizar (sin } \\
\text { objetivo de producto) }\end{array}$ & 1,04 & 12,62 & 10,91 & -0.03 & -2.50 & -0.55 & $-3.08 \%$ \\
\hline
\end{tabular}

Pérdidas de bienestar asociadas a diferentes arreglos de política monetaria en Costa Rica (Porcentaje del consumo en Estado Estacionario)

Fuente: elaboración propia.

Las simulaciones indican que con respecto al escenario base, los arreglos de política de tipo de cambio fijo y flotación administración presentan los mayores costos sociales.

El valor de $\delta_{\mathrm{e}}$ es calibrado en la magnitud de -0.54, haciendo uso de regresiones de mínimos cuadrados ordinarios utilizando los datos macroeconómicos de Costa Rica; como parte de los ejercicios de simulación se asume un parámetro de manera arbitraria de magnitud 1 para realizar comparaciones; los resultados indican que los costos sociales incrementan entre mayor sea la ponderación que el Banco Central le atribuya al objetivo de mantener la estabilidad cambiaria. 


\section{Conclusiones}

Este ejercicio econométrico es de gran valor, ya que permite simular la economía en diversas situaciones para poder analizar las trayectorias de las variables en diferentes ámbitos de toma de decisiones como, por ejemplo: política fiscal, comercial, monetaria, etc. En este ejercicio se detectaron rigideces de precios importantes, la estimación del parámetro de Calvo $(\omega)$, que indica rigidez en los precios, se estima en 0,6203 lo que significa que una empresa cambia sus precios, en promedio, cada 7 meses con 13 días; además, se encontró que las expectativas de inflación presentan desvíos sistemáticos, lo que evidencia un proceso lento en el aprendizaje de los agentes en la generación de expectativas. Estas condiciones indican que las acciones de la política monetaria pueden tener efectos reales en el corto plazo, determinan la dinámica de ajuste de la economía, y explica gran parte de la persistencia encontrada en las funciones de impulso-respuesta.

Los parámetros estimados que representan la política monetaria muestran que el objetivo de producción $\left(\delta_{y}\right)$ al igual que el parámetro de objetivo de inflación $\left(\delta_{\pi}\right)$ son mayores a los priors seleccionados; el relativo de los parámetros prior de política monetaria $\left(\delta_{\pi} / \delta_{y}\right)$, que representan el estándar internacional, es de una magnitud de $11.53 \mathrm{y}$, para Costa Rica, el valor es 8.6; lo que indica que el Banco Central de Costa Rica da un mayor peso al objetivo de estabilizar el ciclo económico. Asimismo, en la Regla de Taylor estimada predomina el elemento inercial sobre los objetivos de política monetaria lo que indica un bajo activismo a la hora de realizar política por parte del Banco Central de Costa Rica.

Las simulaciones de efectos sobre el bienestar de posibles regímenes de política monetaria indican que con respecto al escenario base, los escenarios de tipo de cambio fijo y flotación administrada presentan los mayores costos sociales. Existe evidencia que indica que los costos se incrementan entre mayor sea la importancia relativa del objetivo de política de mantener la estabilidad cambiaria. El arreglo de política que provoca los menores costos sociales es el caso de metas de inflación "puras" 22 (o sin objetivo de estabilización del producto), lo que indica que en el largo plazo la estabilización de la inflación implica la estabilización en el ciclo económico y neutralidad monetaria, bajo el enfoque del modelo de Lucas (1972).

Como recomendaciones de Política Económica, lo siguiente: el Banco Central de Costa Rica debe avanzar a un régimen de Metas de Inflación "Puras" (sin objetivo sobre el nivel de producción) para minimizar las pérdidas sociales de bienestar causadas por la volatilidad del ciclo económico y la inflación. En una transición "ordenada" a un régimen de Metas de Inflación es muy probable que se asuma el régimen de Flotación Administrada del Tipo de Cambio por un tiempo (no acotado); por lo tanto, debido a las pérdidas de bienestar que esto causa (Tabla 5), el Banco

22 Es importante tomar en consideración que implícitamente no son puras ya que en el corto plazo se puede priorizar la estabilización del producto sobre la inflación.

24

Marco Vinicio Valerio Berrocal

(c) (P) 8 (2)

Revista Economía y Sociedad by Universidad Nacional is licensed under a CreativeCommons Reconocimiento-NoComercial- 
Central de Costa Rica no debe tener reglas de intervencion muy rígidas en el mercado cambiario. Es decir, no debe renunciar al objetivo de inflación con el fin de reducir la volatilidad del tipo de cambio. La velocidad en que los agentes ajustan sus expectativas puede aumentar si el Banco Central de Costa Rica provee información pertinente y accesible sobre sus metas y cumplimiento de objetivos; mejorar la efectividad de este mecanismo es fundamental para generar credibilidad y anclar la expectativa de inflación a las metas del Banco Central.

Se recomienda en futuras investigaciones extender la capacidad explicativa del modelo para evaluar determinantes de la transmisión de la política monetaria que fueron abstraidos para acotar el análisis. El grado de dolarización de la economía reduce la efectividad de todos los mecanismos de trasmisión debido a que las modificaciones a la base monetaria que realizan los bancos centrales y de tasa de interés son denominadas en moneda nacional, por lo tanto en una economía parcialmente dolarizada; la efectividad de la política monetaria es parcialmente efectiva. La polititica fiscal determina la efectividad de los mecanismos de transmisición de la política monetaria. La dominancia fiscal provoca que los bancos centrales sigan una política expansionista con el fin de financiar al fisco y elevar la producción por encima del nivel potencial, lo que causa pérdidas de eficiencia y costos sociales importantes.

\section{Referencias}

Calvo, G. (1983). Staggered prices in a utility-maximizing frameword. Journal of Monetary Economics, 12(3), 383-398. doi: http://dx.doi.org/10.1016/0304-3932(83)90060-0

Christiano, L., Trabant, M., \& Walentin, K. (2010). DSGE Models for Monetary Policy. En M, Woodford, \& B, Friedman, Handbook of Monetary Economics. 3, 285-367. Recuperado de http://www.sciencedirect.com/science/handbooks/15734498

Clarida, R., Galí, J., \& Gertler, M. (1999). The Science of Monetary Polcy: A new Keynesian Perspective. Journal of Economic Literature, 37 (4), 1661-1707. doi: http://dx.doi.org/10.1257/jel.37.4.1661

Cuadrado, J. (2006). Política económica: Objetivos e instrumentos. Recuperado de https://books.google.co.cr/books/about/Pol\%C3\%ADtica econ\%C3\%B3mica.html?id=Mbs5nQA ACAAJ\&redir esc $=y$

Del Negro, M., Schorfheide, F., Smets, F., \& Wouters, R. (2007). On the fit of New Keynesian Models. Journal of Business \& Economic Statistics, 25 (2), 123-143. doi: http://dx.doi.org/10.1198/073500107000000016 
Escudé, G. (2010). Modelos de Equilibrio General Dinámico y Estocástico (EGDE): Una introducción (Documento de Trabajo N. 47). Recuperado del sitio web del Banco Central de Argentina: http://www.bcra.gov.ar/pdfs/investigaciones/WP 47 2010e.pdf

Dhrymes, P. (1970). Econometrics Statistical foundations and aplications. Berlin: SpringerVerlag.

Galí, J. (2008). Monetary Policy, Inflation, and the Business Cycle: An Introduction to the New Keynesian Framework. Recuperado de http://press.princeton.edu/titles/10495.html

Galí, J., \& Monacelli, T. (2002). Monetary Policy and Exchange Rate Volatility in a Small Open Economy (Working Paper 8905). Recuperado del sitio de internet National Bureau of Economic Research: http://dx.doi.org/10.3386/w8905

Galí, J., \& Monacelli, T. (2005). Monetary Policy and Exchange Rate Volatility in a Small Open Economy. The Review of Economic Studies, 72 (3), 707-734. doi: http://dx.doi.org/10.1111/i.1467-937X.2005.00349.x

García, C., \& González, W. (2007). Efectividdad de la Política Monetaria en Algunas Economías Latinoamericanas. Recuperado de https://www.econbiz.de/Record/efectividad-de-lapol\%C3\%ADtica-monetaria-en-algunas-econom\%C3\%ADas-latinoamericanas-garciacarlos/10005673218/Description\#tabnav

Hansen, L., \& Sargent, T. (1993). Seasonal and aproximation errors in rational expectation models. Journal of Econometrics, 55(1-2), 21-55. doi: http://dx.doi.org/10.1016/03044076(93)90003-N

Hicks, J. (1974). La economía del bienestar. México: Fondo de Cultura Económica.

Learmer, E. (1991). A Bayesian Perspective on Inference from Macroeconomic Data. The Scandinavian Journal of Economics, 93 (2), 225-248. Recuperado de https://www.jstor.org/stable/3440330

León J., \& Valerio, M. (2014). Persistencia Inflacionaria en Costa Rica: Precios de Servicios y Regulados. Ciencias Económicas, 32 (2), 155-187. doi: http://dx.doi.org/10.15517/rce.v32i2.17263

Liberati, D. (2012). Interest Rate Pass-Through and Credit Spread in New Keynesian Models Theoretical and Empirical Relevance (Doctoral Thesis). University of Rome. Recuperado de http://padis.uniroma1.it/handle/10805/1987

26

Marco Vinicio Valerio Berrocal

(c) (i) (2)(2)

Revista Economía y Sociedad by Universidad Nacional is licensed under a CreativeCommons Reconocimiento-NoComercialCompartirlgual 4.0 Internacional License. Creado a partir de la obra en http://www.revistas.una.ac.cr/index.php/economia 
Lucas, R. (1976). Econometric Policy Evaluation: A critique. Journal of Monetary Economics, 1, 19-46. Recuperado de http://www.sciencedirect.com/science/journal/01672231/1

Lucas, R. (1972). Expectations and the Neutrality of Money. Journal of Economic Theory, 4 (2), 103-124. doi: http://dx.doi.org/10.1016/0022-0531(72)90142-1

Mohamed, B., \& Sales, M. (2015). Credit imperfections, labor market frictions and unemployment: a DSGE approach. Recuperado de https://hal.inria.fr/hal$\underline{01082491 / \text { document }}$

Muñoz, E., y Tenorio, E. (2008). El Modelo Macreoeconómico de Proyección Trimestral del Banco Central de Costa Rica en la Transición a la Flexibilidad del Tipo de Cambio. Recuperado de http://www.bccr.fi.cr/investigacioneseconomicas/politicamonetariaeinflacion/Modelo Macroe conomico proyeccion trimestral transicion flexibilidad cambiaria.pdf

Segura, C., \& Vásquez, J, (2011). Estimación del parámetro de suavizamiento del filtro de Hodrick y Prescott para Costa Rica (DEC-DIE-DT 006-2011). Recuperado de http://www.bccr.fi.cr/investigacioneseconomicas/metodoscuantitativos/Estimacion del param etro de suavizamiento del filtro de Hodrick y Prescott para CR.pdf

Sims, C. (1980). Macroeconomics and Reality. Econometrica, 48 (1), 1-48. Recuperado de http://www.jstor.org/stable/1912017

Tinbergen, J. (1956). Economic policy: Principles and Design. Recuperado de https://books.google.co.cr/books/about/Economic Policy.html?id=Yq4QAAAAIAAJ\&redir esc=y

Taylor, J. (1993). Discretion versus policy rules in practice. Carnegie-Rochester Conference Series on Public Policy, Stanford University, 39 (1), 195-214. Recuperado de http://web.stanford.edu/ johntayl/Onlinepaperscombinedbyyear/1993/Discretion versus Poli cy Rules in Practice.pdf

Winkelried, D. (2013). Modelo de Proyección Trimestral del BCRP Actualización y Novedades. Revista de Estudios Económicos, 26, 9-60. Recuperado de http://www.bcrp.gob.pe/docs/Publicaciones/Revista-Estudios-Economicos/26/ree-26winkelried.pdf

Woodford, M. (2001). Inflation stabilization and welfare (Working Paper 8071). Recuperado de National Bureau of Economic Research: http://dx.doi.org/10.3386/w8071

Woodford, M. (2003). Interest and prices: Foundations of a theory of monetary policy. Recuperado de http://press.princeton.edu/titles/7603.html 
Anexos
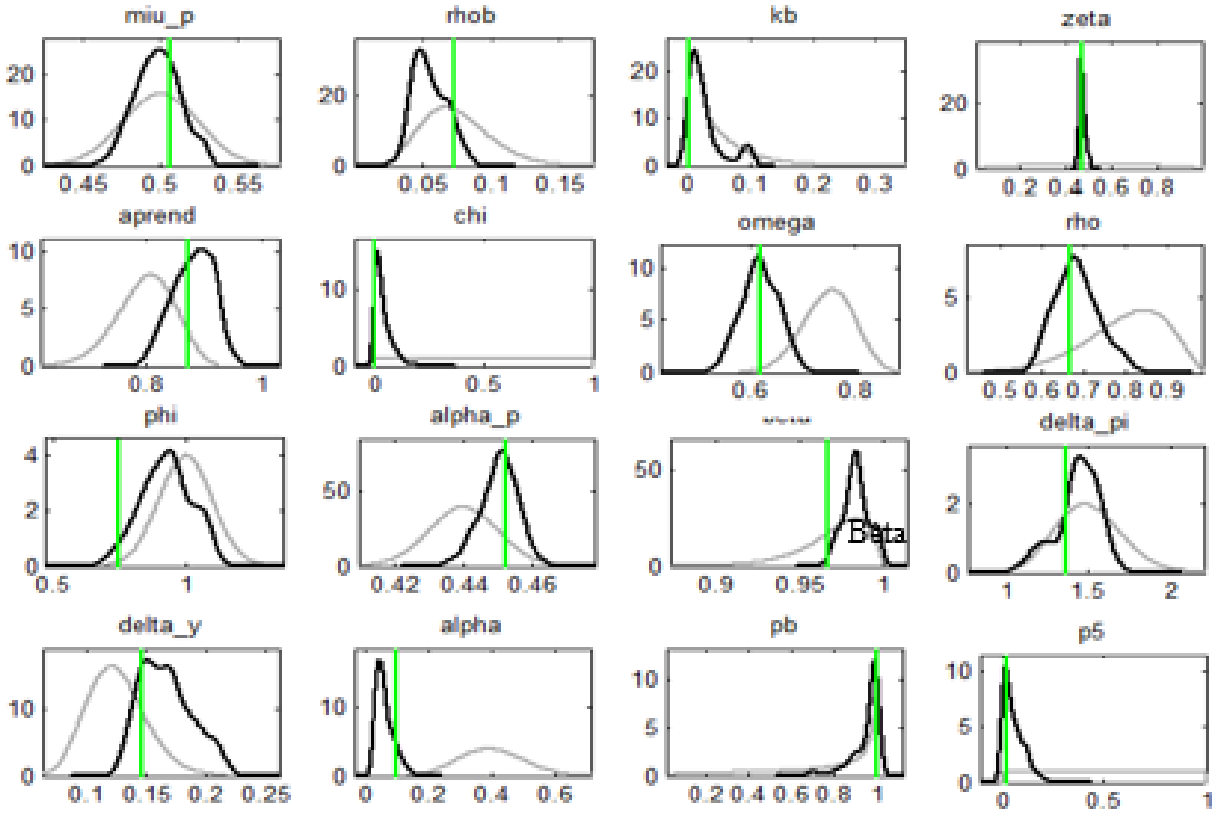

alpha
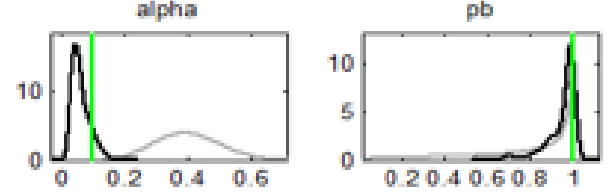

p5
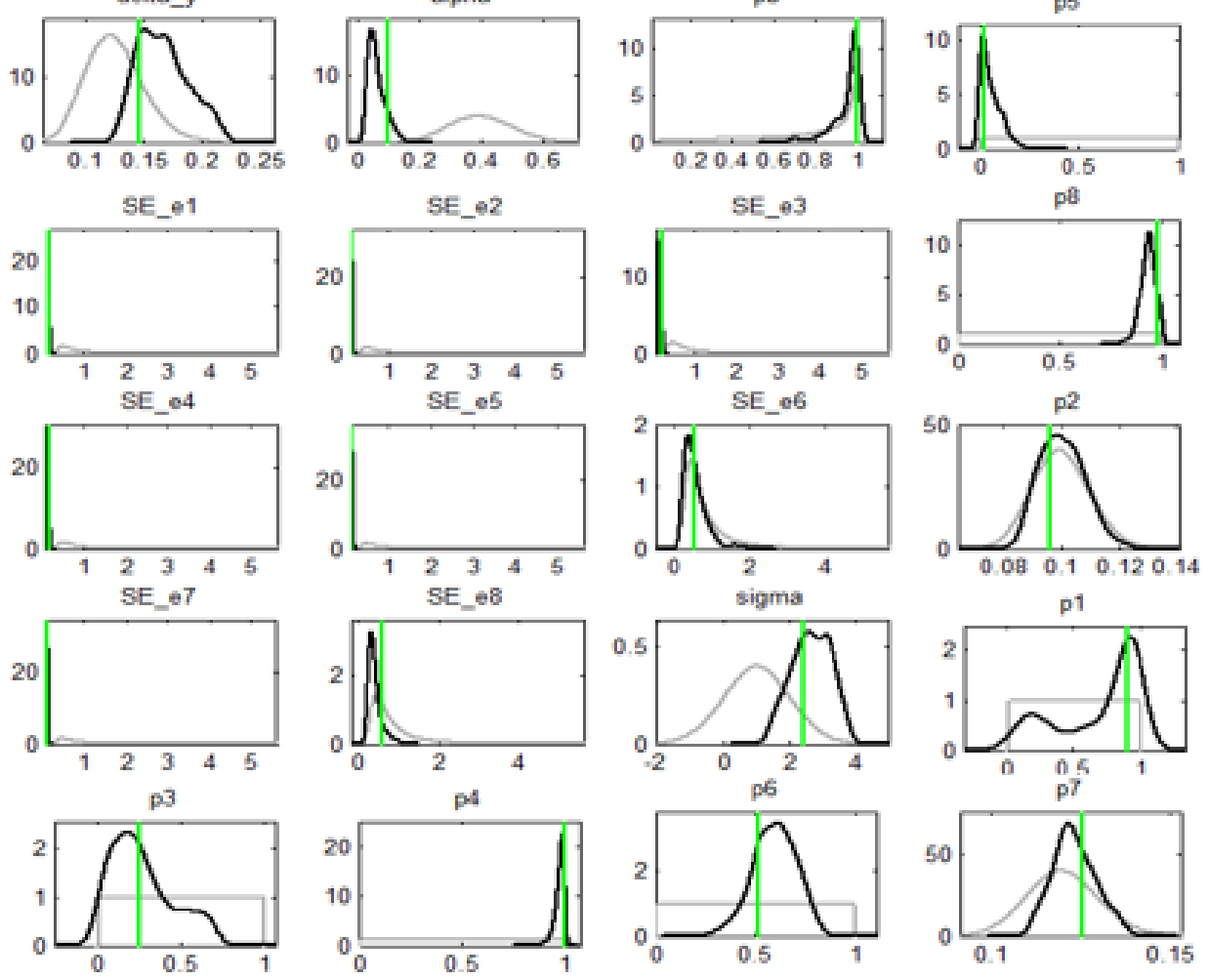

Figura 2. Distribuciones Posterior. Fuente: Elaboración propia. Nota: Valor del parámetro en el eje de las abscisas, probabilidad de ocurrencia asociado al parámetro en el eje de las ordenadas.

28

\section{(c) (i) (2)(2)}

Revista Economía y Sociedad by Universidad Nacional is licensed under a CreativeCommons Reconocimiento-NoComercial- 


\section{Código MATLAB/DYNARE}

//The evaluation of Monetary Policy Transmission Mechanism in a DSGE Model:

//The Case of Costa Rica (2006-2014).

//linearized model

//Lic.Marco Vinicio Valerio Berrocal

//mail:markxsw@gmail.com

var sh8 w_n n pi_p mc_w w pi_w hh rl theta_crd y yqf yf x pi_h mc pi e q psi s y_ex ...

pi_ex r_exs_f v_b p_bh q_bl sh1 sh2 pi_exp sh3 sh4 sh5 sh6 sh7;

varexo e1 e2 e3 e4 e5 e6 e7 e8;

parameters p8 sigma phi alpha_p chi_ex chi beta omega omega_w rho delta_pi delta_y...

alpha beta_ex kappa_ex sigma_ex rho_ex delta_pi_ex delta_y_ex miu_p rhob kb RD W N RL...

pb zeta p1 p2 p3 p4 p5 p6 p7 aprend;

beta_ex=0.99;

kappa_ex $=0.21$;

sigma_ex $=1$;

rho_ex $=0.87$;

delta_pi_ex=1.43;

delta_y_ex $=0.04$;

chi_ex=0.76;

$\mathrm{RD}=1.0101$;

$\mathrm{W}=1$;

$\mathrm{N}=1$;

$\mathrm{RL}=1.0401$;

omega_w $=0.5$;

model(linear);

pi_ex=beta_ex*pi_ex $(+1)+$ kappa_ex*y_ex;

(y_ex-chi_ex*y_ex $(-1))=\left(y \_e x(+1)-c h i \_e x * y \_e x\right)-(1 / \operatorname{sigma} e x)^{*}(r$ ex-pi_ex $(+1))$;

r_ex=r_ex $(-1) *$ rho_ex+(1-rho_ex $)^{*}($ delta_pi_ex*pi_ex+delta_y_ex*y_ex)+sh7;

$\mathrm{hh}=\mathrm{x}-\mathrm{chi}^{*} \mathrm{x}(-1)$;

$\mathrm{hh}=\mathrm{hh}(+1)-(1 /$ sigma $) *($ rd-pi_exp $)$;

pi_exp=pi_exp $(-1) *$ aprend $+(1$-aprend $) *$ pi $(+1)+$ sh5;

$\mathrm{rl}=\left(\left((((1-a)\right.\right.$ pha_p $\left.) * z e t a) /\left(\mathrm{RL} *\left(-a l p h a \_p+z e t a\right)\right)\right) *(\mathrm{RD}+(((1-\mathrm{rhob}) * \mathrm{pb}) /(\mathrm{N} * \mathrm{~W})) *(\mathrm{~kb} / \quad(((\mathrm{kb} * \mathrm{RD} *(1-(1-\mathrm{rhob}) *$ beta $\left.\left.)) /(\mathrm{RL}-\mathrm{RD}) * \mathrm{~W} * \mathrm{~N})) \quad))\right)\right)^{*} \mathrm{rd}(-$

$1)+\left(\left(\left(\left(1-a l p h a \_p\right) * z e t a\right) /\left(R L^{*}\left(-a l p h a \_p+z e t a\right)\right)\right) *\left(((1-r h o b) * p b) /\left(N^{*} W\right)\right) *(k b /\right.$

$\left(\left(\left(k b^{*} R D^{*}(1-(1-r h o b) *\right.\right.\right.$ beta $\left.\left.\left.)\right) /(R L-R D) * W^{*} N\right)\right)$

)$) *($ theta_c $(+1)+w+n-r d(+1)-p i(+1))+$ sh6;

yqf=yf+((1-alpha_p)/(1+(1-alpha_p $)^{*}($ sigma-1)+phi) $) * r l$;

$\mathrm{yf}=\operatorname{sh} 1 *\left((p h i+1) /\left(\left(1-a l p h a \_p\right) *(\operatorname{sigma}-1)+\right.\right.$ phi+1) $)-\left((1-\right.$ alpha_p $) /\left(\left(1-a l p h a \_p\right) *(\right.$ sigma-1)+phi+1) $) * r l ;$

$\mathrm{mc}=\mathrm{rl}+\left(\left(\left(1-a l p h a \_p\right)^{*}(\operatorname{sigma}-1)+\text { phi+1) } /(1-\text { alpha_p })\right)^{*}\left((1 /(1-\mathrm{chi}))^{*}(\mathrm{hh})+\right.\right.$ alpha*s $)$;

$\mathrm{x}=\mathrm{y}$-yqf;

pi_p=beta*pi_p(+1)+(((1-omega*beta)*(1-omega))/(omega) $) * m c ;$

rd=rho*rd(-1)+(1-rho)*(delta_pi*(pi)+delta_y*x)+sh2;

$x$-chi*x(-1)=y_ex-chi_ex*y_ex(-1)+(1/sigma $)^{*}(1-c h i) * q$;

pi=pi_h+alpha*(s-s(-1));

$q=p s i+(1-a l p h a) * s$;

psi=e+pi_ex-(1-alpha)*pi;

$s=s(-1)-p i+p i \_e x+e+s h 3$;

theta_c=s_f-v_b;

$h=m i u \_p * v \_b+\left(1-m i u \_p\right)^{*} s \_f-s h 4$;

p_b=h-s_f;

q_b $=h-v_{-}$b;

$s_{-} f=-((1-r h o b) *(p b /((r h o b+p b)-r h o b * p b)) /(1-(1-r h o b) *(p b /((r h o b+p b)-r h o b * p b)))) * I(-1)$;

$\mathrm{I}=(1-\mathrm{rhob}) * \mathrm{I}(-1)+\mathrm{rhob} * \mathrm{~h}$;

$\left(\left(R D^{*} k b\right) /\left(\left((k b * R D *(1-(1-r h o b) * b e t a)) /(R L-R D) * W^{*} N\right)\right)\right)^{*}\left(r d-q \_b\right)=R L^{*} W^{*} N *(r l(-1)+w+n)-R D * W * N *(r d+w+n)+((1-r h o b) * b e t a *((R D * k b) /$ $\left(\left((k b * R D *(1-(1-r h o b) *\right.\right.$ beta $\left.\left.\left.\left.)) /(\mathrm{RL}-\mathrm{RD}) * \mathrm{~W}^{*} \mathrm{~N}\right)\right)\right)\right)^{*}\left(\mathrm{rd}(+1)-\mathrm{q} \_\mathrm{b}(+1)-\operatorname{sigma} *(\mathrm{hh}(+1)-\mathrm{hh})\right)$;

pi_h=(1/((((1-omega_w*beta)*(1-omega_w))/(omega_w) $)+(((1-$ omega*beta)*(1-omega) $) /($ omega $))))^{*}((((1-$ omega_w*beta)* $(1-$

omega_w) $) /($ omega_w $))^{*}$ pi_w+((((1-omega*beta)*(1-omega))/(omega) $\left.)\right)^{*}$ pi_p);

pi_w=beta*pi_w(+1)+(((1-omega_w*beta)*(1-omega_w $)) /($ omega_w $))^{*}$ mc_w;

mc_w $=$ w-(sigma+phi/(1-alpha_p) $)^{*} x$;

$w(-1)=w+p i \_w-p i \_p-\left(w \_n-w \_n(-1)\right)-s h 8$;

w_n $\left.=\log \left(1-a \mid p h a \_p\right)+\left(\left((1-a) p h a \_p *\left((1+p h i) /\left(\operatorname{sigma*}\left(1-a l p h a \_p\right)+a l p h a \_p+p h i\right)\right)\right)\right) /\left(1-a l p h a \_p\right)\right) * \operatorname{sh} 1$;

Marco Vinicio Valerio Berrocal

(c) 9 (5)

Revista Economía y Sociedad by Universidad Nacional is licensed under a CreativeCommons Reconocimiento-NoComercial-

Compartirlgual 4.0 Internacional License.

Creado a partir de la obra en http://www.revistas.una.ac.cr/index.php/economia 


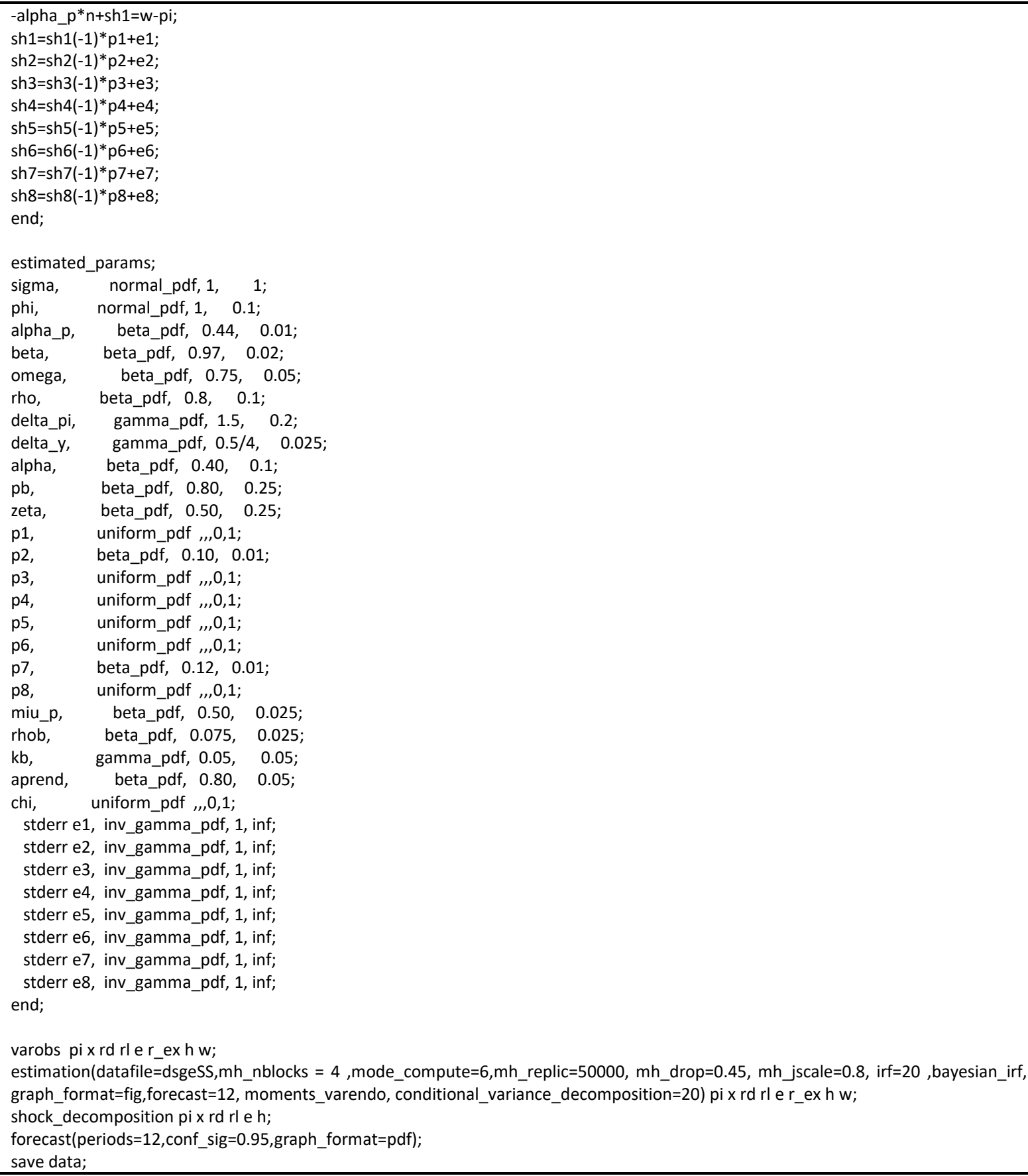

30

Marco Vinicio Valerio Berrocal

(cc) (i) 8 (O)

Revista Economía y Sociedad by Universidad Nacional is licensed under a CreativeCommons Reconocimiento-NoComercial- 


\section{Modelo Completo Log-Linearizado}

Curva IS dinámica;

$$
\hat{y}_{t}-\chi \hat{y}_{t-1}=E_{t}\left(\hat{y}_{t+1}-\chi \hat{y}_{t}\right)-\frac{1}{\sigma}\left(r_{t}{ }^{1}-\pi_{t}{ }^{e}\right)
$$

Formación de expectativas;

$$
\pi_{\mathrm{t}}^{\mathrm{e}}=\rho_{\text {aprend }} \pi_{\mathrm{t}-1} \mathrm{e}+\left(1-\rho_{\text {aprend }}\right) \mathrm{E}_{\mathrm{t}}\left(\pi_{\mathrm{t}+1}\right)
$$

Curva de Phillips;

$$
\pi_{h, t}=\beta E\left(\pi_{h, t+1}\right)+\frac{(1-\omega)(1-\omega \beta)}{\omega} \mathrm{mc}_{\mathrm{t}}
$$

Costos Marginales;

$$
\mathrm{mc}_{\mathrm{t}}=\mathrm{r}_{\mathrm{t}}^{\mathrm{l}}+\left(\frac{\left(1-\alpha^{\prime}\right)(\sigma-1)+\varphi+1}{1-\alpha^{\prime}}\right) \frac{1}{1-\chi}\left(\hat{\mathrm{y}}_{t}-\chi \hat{\mathrm{y}}_{t-1}\right)+\alpha s_{t}
$$

Brecha del Producto;

$$
\widehat{y_{t}}=y_{t}-y_{t}^{q f}
$$

Producción de precios quasi flexibles;

$$
\mathrm{y}_{\mathrm{t}}^{\mathrm{qf}}=\mathrm{y}_{\mathrm{t}}^{\mathrm{f}}+\left(\frac{1-\alpha^{\prime}}{\left(1-\alpha^{\prime}\right)(\sigma-1)+\varphi+1}\right) \mathrm{r}_{\mathrm{t}}^{\mathrm{l}}
$$

Producción de precios flexibles;

$$
\mathrm{y}_{\mathrm{t}}^{\mathrm{f}}=\left(\frac{1+\varphi}{\left(1-\alpha^{\prime}\right)(\sigma-1)+\varphi+1}\right) \mathrm{a}_{\mathrm{t}}-\left(\frac{1-\alpha^{\prime}}{\left(1-\alpha^{\prime}\right)(\sigma-1)+\varphi+1}\right) \mathrm{r}_{\mathrm{t}}^{\mathrm{l}}
$$

Determinación de la tasa de interés activa;

$$
\mathrm{r}_{\mathrm{t}}^{\mathrm{l}}=\left(\frac{\left(1-\alpha^{\prime}\right) z}{\left(R^{L}\left(-\alpha^{\prime}+z\right)\right)}\right)\left(R^{D}+\frac{\left(1-\rho^{B}\right) p^{B}}{N w} \frac{k^{B}}{q^{B}}\right) r_{t}^{d}+\left(\frac{\left(1-\alpha^{\prime}\right) z}{\left(R^{L}\left(-\alpha^{\prime}+z\right)\right)}\right)\left(\frac{\left(1-\rho^{B}\right) p^{B}}{N w} \frac{k^{B}}{q^{B}}\right)\left(\widehat{\theta_{\mathrm{t}}^{\mathrm{c}}}+\widehat{\mathrm{w}_{\mathrm{t}}}-r_{t+1}^{d}-\pi_{\mathrm{t}+1}\right)
$$

Regla de Política Monetaria;

$$
r_{t}^{d}=\rho r_{t-1}^{d}+(1-\rho)\left(\delta_{\pi} E\left(\pi_{t+1}\right)+\delta_{y} \hat{y}_{t}\right)
$$

Tipo de Cambio Real;

$$
\mathrm{q}_{\mathrm{t}}=\psi_{\mathrm{t}}+(1-\alpha) \mathrm{s}_{\mathrm{t}}
$$

Desvíos de la Ley de un solo precio;

$$
\Delta \psi_{\mathrm{t}}=\mathrm{e}_{\mathrm{t}}-\mathrm{e}_{\mathrm{t}-1}-\pi *_{\mathrm{t}}+\alpha \pi_{\mathrm{t}}
$$

Términos de Intercambio;

$$
\mathrm{s}_{\mathrm{t}}=\left(\mathrm{r}_{\mathrm{t}}^{*}-\mathrm{E}_{\mathrm{t}}\left\{\pi_{\mathrm{t}+1}^{*}\right\}\right)-\left(\mathrm{r}_{\mathrm{t}}^{\mathrm{d}}-\mathrm{E}_{\mathrm{t}}\left\{\pi_{\mathrm{H}, \mathrm{t}+1}\right\}\right)+\mathrm{E}_{\mathrm{t}}\left\{\mathrm{s}_{\mathrm{t}+1}\right\}
$$

Relación de Arrastre en la economía local y el mundo;

$$
y=y_{t}^{*}+\left(\frac{1}{\sigma}\right) q_{t}
$$

Identidades del Mercado Crediticio;

$$
\begin{aligned}
\widehat{\mathrm{P}_{\mathrm{t}}^{\mathrm{b}}} & =\widehat{\mathrm{H}}_{\mathrm{t}}-\widehat{\mathrm{s}}_{\mathrm{t}}^{\mathrm{F}} \\
\widehat{\mathrm{q}}_{\mathrm{t}}^{\mathrm{b}} & =\widehat{\mathrm{H}}_{\mathrm{t}}-\widehat{\mathrm{V}}_{\mathrm{t}}^{\mathrm{b}} \\
\widehat{\hat{\theta}}_{\mathrm{t}}^{\mathrm{c}} & =\widehat{\mathrm{s}}_{\mathrm{t}}^{\mathrm{F}}-\widehat{\mathrm{V}}_{\mathrm{t}}^{\mathrm{b}}
\end{aligned}
$$




$$
\begin{aligned}
\widehat{\mathrm{H}}_{\mathrm{t}} & =\left(1-\mu^{\prime}\right) \hat{\mathrm{S}}_{\mathrm{t}}^{\mathrm{B}}+\mu^{\prime} \hat{\mathrm{s}}_{t}^{F} \\
\hat{\mathrm{S}}_{\mathrm{t}}^{\mathrm{B}} & =-\frac{\left(1-\rho^{\mathrm{B}}\right) \mathrm{L}^{\mathrm{B}}}{\mathrm{s}^{\mathrm{F}}} \hat{\mathrm{L}}_{\mathrm{t}-1}^{\mathrm{B}} \\
\hat{\mathrm{L}}_{\mathrm{t}}^{\mathrm{B}} & =\left(1-\rho^{\mathrm{B}}\right) \hat{\mathrm{L}}_{\mathrm{t}-1}^{\mathrm{B}}+\rho^{\mathrm{B}} \widehat{\mathrm{H}}_{\mathrm{t}}
\end{aligned}
$$

Ecuación de creación de créditos;

$$
\frac{R^{d} k^{B}}{q^{B}}\left(r_{t}^{d}-\hat{q}_{t}^{B}\right)=\left(\begin{array}{llll}
w & N & R^{L}
\end{array}\right) r_{t-1}^{1}-\left(\begin{array}{lll}
w & N & R^{D}
\end{array}\right) r_{t}^{d}+\left(1-\rho^{B}\right) \beta \frac{R^{d} k^{B}}{q^{B}}\left(r_{t+1}^{d}-\hat{q}_{t+1}^{B}\right)
$$

Bloque externo;

$$
\begin{gathered}
\hat{\mathrm{y}}^{*}{ }_{\mathrm{t}}-\chi^{*} \hat{\mathrm{y}}^{*}{ }_{\mathrm{t}-1}=\mathrm{E}_{\mathrm{t}}\left(\hat{\mathrm{y}}^{*}{ }_{\mathrm{t}+1}-\chi^{*} \hat{\mathrm{y}}^{*}{ }_{\mathrm{t}}\right)-\frac{1}{\sigma^{*}}\left(\mathrm{r}_{\mathrm{t}}{ }^{*}-\mathrm{E}\left(\pi_{\mathrm{t}+1}{ }^{*}\right)\right) \\
\pi^{*}{ }_{\mathrm{t}}=\kappa\left(\widehat{\mathrm{y}_{\mathrm{t}}}\right)+\beta \mathrm{E}\left(\pi^{*}{ }_{\mathrm{t}+1}\right)+\varepsilon_{\mathrm{t}} \\
\mathrm{r}_{\mathrm{t}}^{*}=\Omega^{*} \mathrm{r}_{\mathrm{t}-1}^{* 1}+\left(1-\Omega^{*}\right)\left(\phi_{\pi} \mathrm{E}\left(\pi^{*}{ }_{\mathrm{t}+1}\right)+\phi_{\mathrm{y}} \hat{\mathrm{y}}_{\mathrm{t}}^{*}\right)
\end{gathered}
$$

Costo marginal salarial;

$$
\widehat{m c}_{t}^{w}=\widehat{w}_{t}-\left(1+\frac{\varphi}{\left(1-\alpha^{\prime}\right)}\right) \widehat{y}_{t}
$$

Dinámica de ajuste de los salarios;

$$
\widehat{w}_{t-1}=\widehat{w}_{t}-\pi_{t}^{w}+\left(\bar{w}_{t}-\bar{w}_{t-1}\right)
$$

Demanda de trabajo

$$
-\alpha n_{t}+a_{t}=\widehat{w}_{t}-\pi_{t}
$$

Salarios de precios flexibles

$$
\bar{w}_{t}=\log \left(1-\alpha^{\prime}\right)+\left(\left(\frac{1-\frac{1+\varphi}{\sigma\left(1-\alpha^{\prime}\right)+\alpha^{\prime}+\varphi}}{1-\alpha^{\prime}}\right)\right) a_{t}
$$

Determinación de la Inflación Doméstica

$$
\pi_{h, t}^{\prime}=\frac{1}{\lambda_{p}+\lambda_{w}}\left(\lambda_{p} \pi_{h, t}+\pi_{t}^{w} \lambda_{w}\right)
$$

Determinación de la Inflación General

$$
\pi_{t}=\pi_{h, t}^{\prime}+(1-\alpha) \pi_{t}^{*}
$$

Inflación por determinación salarial

$$
\pi_{t}^{w}=\beta E_{t}\left(\pi_{t+1}^{w}\right)+\left(\frac{\left(1-\omega_{W}\right)\left(1-\beta \omega_{W}\right)}{\omega_{W}}\right) \widehat{m c}_{t}^{w}
$$

\section{Problemas de Maximización de los Agentes del Modelo}

Hogares

$$
\begin{gathered}
\text { Máx } E_{0} \sum_{t=0}^{\infty} \beta^{t}\left(\frac{1}{1-\sigma}\left(\frac{C_{t}}{C_{t-1}} C_{t-1}^{(1-\chi)}\right)^{1-\sigma}-\frac{N_{t}^{1+\varphi}}{1+\varphi}\right) \\
\text { st. } \quad P_{t} C_{t}+E_{t}\left(Q_{t+1} D_{t+1}\right) \leq D_{t}+W_{t} N_{t}+T_{t}
\end{gathered}
$$

32 
Economía y Sociedad, Vol. 21, № 50 julio-diciembre 2016, pp. 1-33

Fijación de Salarios

$$
\begin{gathered}
\operatorname{máx}_{\mathrm{w}} \sum_{\mathrm{k}=0}^{\infty}\left(\omega_{W} \beta\right)^{\mathrm{k}} \mathrm{E}_{\mathrm{t}}\left\{\frac{1}{1-\sigma}\left(\frac{\mathrm{C}_{\mathrm{t}}}{\mathrm{C}_{\mathrm{t}-1}} \mathrm{C}_{\mathrm{t}-1}^{(1-\chi)}\right)^{1-\sigma}-\frac{\mathrm{N}_{\mathrm{t}}^{1+\varphi}}{1+\varphi}\right\} \\
\text { st. } \mathrm{N}(\mathrm{i})_{\mathrm{t}}=\left(\frac{\mathrm{W}(\mathrm{i})_{\mathrm{t}}}{\mathrm{W}_{\mathrm{t}}}\right)^{-\varepsilon_{w}} \mathrm{~N}_{\mathrm{h}, \mathrm{t}}
\end{gathered}
$$

Empresas

$$
\begin{gathered}
\operatorname{máx}_{\bar{P}_{\mathrm{H}, \mathrm{t}}} \sum_{\mathrm{k}=0}^{\infty}(\theta \beta)^{\mathrm{k}} \mathrm{E}_{\mathrm{t}}\left\{\frac{\lambda_{\mathrm{t}+1}}{\lambda_{\mathrm{t}}}\left[\frac{\mathrm{P}_{\mathrm{H}, \mathrm{t}}^{*}}{\mathrm{P}_{\mathrm{H}, \mathrm{t}+\mathrm{k}}}-m c_{\mathrm{t}+1}\right] \mathrm{Y}(\mathrm{i})_{\mathrm{h}, \mathrm{t}+1}\right\} \\
\text { st. } \mathrm{Y}(\mathrm{i})_{\mathrm{h}, \mathrm{t}}=\left(\frac{\mathrm{P}(\mathrm{i})_{\mathrm{h}, \mathrm{t}}}{\mathrm{P}_{\mathrm{h}, \mathrm{t}}}\right)^{-\varepsilon} \mathrm{Y}_{\mathrm{h}, \mathrm{t}}
\end{gathered}
$$

\begin{tabular}{|c|c|}
\hline $\bar{w}_{t}$ & Salario de estado estacionario \\
\hline$n_{t}$ & Horas de trabajo \\
\hline$\pi_{h, t}^{\prime}$ & Inflación domestica \\
\hline$\widehat{m c} c_{t}^{w}$ & Brecha Costos Marginales Salariales \\
\hline$\widehat{w}_{t}$ & Brecha de Salarios (Valor menos su tendencia) \\
\hline$\pi_{t}^{w}$ & Inflación por ajustes salariales \\
\hline $\mathrm{r}_{\mathrm{t}}^{1}$ & Tasa de Interés activa \\
\hline$\widehat{\theta_{t}^{c}}$ & Estrujamiento mercado crediticio \\
\hline $\mathrm{r}_{\mathrm{t}}^{\mathrm{d}}$ & Tasa de interés de los depósitos \\
\hline $\mathrm{y}_{t}$ & Producción \\
\hline$y_{t}^{q f}$ & Producción con precios cuasi flexibles \\
\hline$y_{t}^{f}$ & Producción con precios flexibles \\
\hline$\hat{y}_{t}$ & Brecha del producto \\
\hline$\pi_{h, t}$ & Inflación por ajustes en los precios de los bienes \\
\hline $\mathrm{mc}_{\mathrm{t}}$ & Costo Marginal \\
\hline$\pi_{t}$ & Inflación general IPC \\
\hline$e_{t}$ & Tipo de cambio nominal \\
\hline $\mathrm{q}_{\mathrm{t}}$ & Tipo de cambio Real \\
\hline$s_{t}$ & Términos de intercambio \\
\hline$\hat{\mathrm{y}}_{\mathrm{t}}^{*}$ & Brecha del producto de la economía externa \\
\hline$\pi_{t}^{*}$ & Inflación de la economía externa \\
\hline $\mathrm{r}_{\mathrm{t}}^{*}$ & Tasa de interés de la economía externa \\
\hline$\hat{s}_{t}^{b}$ & Proporción del número de empresas que buscan un crédito \\
\hline$\widehat{\mathrm{V}}_{\mathrm{t}}^{\mathrm{b}}$ & Líneas de crédito disponibles \\
\hline$\widehat{\mathrm{P}_{\mathrm{t}}^{\mathrm{b}}}$ & Probabilidad de que ocurra una separación \\
\hline$\widehat{\mathrm{H}}_{\mathrm{t}}$ & Flujo de nuevos créditos \\
\hline$\hat{\mathrm{q}}_{\mathrm{t}}^{\mathrm{b}}$ & Probabilidad de llenar una línea de un crédito \\
\hline$\hat{\mathrm{L}}_{\mathrm{t}}^{\mathrm{B}}$ & saldo de líneas de créditos \\
\hline$\pi_{t}{ }^{e}$ & Expectativas de inflación \\
\hline
\end{tabular}

Bancos

$$
\begin{gathered}
\mathrm{J}_{\mathrm{t}}^{\mathrm{B}}=\max \left[\left(\mathrm{R}_{\mathrm{t}}^{\mathrm{L}}-\mathrm{R}_{\mathrm{t}}^{\mathrm{d}}\right) \mathrm{w}_{\mathrm{t}} \mathrm{N}_{\mathrm{t}} \mathrm{L}_{\mathrm{t}}^{\mathrm{B}}-\mathrm{R}_{\mathrm{t}}^{\mathrm{d}} \mathrm{k}^{\mathrm{B}} \mathrm{V}_{\mathrm{t}}^{\mathrm{B}}+\mathrm{R}_{\mathrm{t}}^{\mathrm{d}} \frac{\mathrm{X}_{\mathrm{t}}}{\mathrm{P}_{\mathrm{t}}}+\beta \mathrm{E}_{\mathrm{t}}\left(\frac{\lambda_{\mathrm{t}+1}}{\lambda_{\mathrm{t}}}\right) \mathrm{J}_{\mathrm{t}+1}^{\mathrm{B}}\right] \\
\text { st. } \quad L_{t}^{B}=\left(1-\rho^{B}\right) L_{t-1}^{B}+q_{t}^{B} V_{t}^{B}
\end{gathered}
$$

\section{Lista de Variables del Modelo}

Fuente: Elaboración propia 